TI 2011-153/1

Tinbergen Institute Discussion Paper

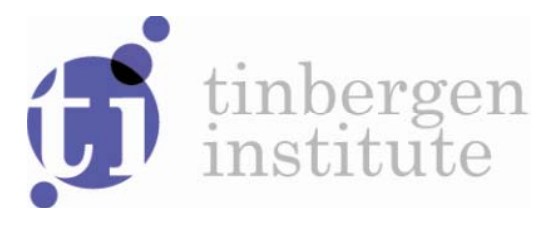

\title{
An Experimental Study of Credible Deviations and ACDC
}

Adrian de Groot Ruiz'

Theo Offerman ${ }^{2}$

Sander Onderstal2

${ }^{1}$ Radboud University Nijmegen;

2 University of Amsterdam. 
Tinbergen Institute is the graduate school and research institute in economics of Erasmus University Rotterdam, the University of Amsterdam and VU University Amsterdam.

More TI discussion papers can be downloaded at http://www.tinbergen.nl

Tinbergen Institute has two locations:

Tinbergen Institute Amsterdam

Gustav Mahlerplein 117

1082 MS Amsterdam

The Netherlands

Tel.: +31(0)205251600

Tinbergen Institute Rotterdam

Burg. Oudlaan 50

3062 PA Rotterdam

The Netherlands

Tel.: +31(0)10 4088900

Fax: $+31(0) 104089031$

Duisenberg school of finance is a collaboration of the Dutch financial sector and universities, with the ambition to support innovative research and offer top quality academic education in core areas of finance.

DSF research papers can be downloaded at: http://www.dsf.nl/

Duisenberg school of finance

Gustav Mahlerplein 117

1082 MS Amsterdam

The Netherlands

Tel.: +31(0)20 5258579 


\section{An Experimental Study of \\ Credible Deviations and ACDC*}

\section{Adrian de Groot Ruiz ${ }^{\mathrm{a}}$, Theo Offerman ${ }^{\mathrm{b}}$ and Sander Onderstal ${ }^{\mathrm{b}}$}

\section{September 12, 2012}

We test the Average Credible Deviation Criterion (ACDC), a stability measure and refinement for cheap talk equilibria introduced in De Groot Ruiz, Offerman \& Onderstal (2012a). ACDC has been shown to be predictive under general conditions and to organize data well in previous experiments meant to test other concepts. In a new experimental setting, we provide the first systematic test of whether and to which degree credible deviations matter for the stability of cheap talk equilibria. Our principal experimental result is that in a setting where existing concepts are silent, credible deviations matter and matter gradually, as predicted by ACDC.

KEYwords: Cheap Talk, Refinement, Selection, Experiment, Neologism Proofness, Announcement Proofness, ACDC

\footnotetext{
${ }^{*}$ We would like to thank seminar participants at Caltech, New York University and the University of Amsterdam. Financial support from the Dutch Science Foundation (NWO-VICI 453-03-606) and from the Amsterdam Research Priority Area Behavioral Economics is gratefully acknowledged.

${ }^{a}$ Department of Economics, Institute for Management Research, Radboud University Nijmegen, P.O, Box 9108, 6500 HK Nijmegen, the Netherlands; a.grootruiz@fm.ru.nl.

${ }^{\mathrm{b}}$ Faculty of Economics and Business, University of Amsterdam, Roetersstraat 11, 1018 WB, the Netherlands; T.J.S.Offerman@uva.nl, onderstal@uva.nl.
} 


\section{Introduction}

Crawford \& Sobel (1982) introduced cheap talk games with asymmetric information, which have found many applications. ${ }^{1}$ Equilibrium selection is important in these games, as they tend to have multiple equilibria with very different levels of information transmission. In this paper, we test the Average Credible Deviation Criterion (ACDC), introduced in De Groot Ruiz, Offerman \& Onderstal (2012a). Many other equilibrium refinements and solution concepts have been proposed for cheap talk games. ${ }^{2}$ These concepts, however, are not able to predict across a wider range of cheap talk games and only a few have been tested experimentally. In contrast, ACDC selects equilbria under general conditions. Its predictions are meaningful in previously analyzed settings and can organize behavior well in existing experiments meant to study other concepts.

ACDC generalizes binary stability criteria based on credible deviations, in particular neologism proofness (Farrell, 1993) and announcement proofness (Matthews, Okuno-Fujiwara \& Postlewaite, 1991). These concepts are based on the observation that out-of-equilibrium messages can have a literal meaning and propose conditions under which messages are credible. ${ }^{3}$ Equilibria that admit such credible deviations are considered unstable. Unfortunately, in many games these criteria do not select a unique equilibrium. The idea behind ACDC is that the credible deviation approach is sound, but that the insistence on a binary distinction between stable and unstable equilibria is problematic. In particular,

\footnotetext{
${ }^{1}$ Applications range from the presidential veto (Matthews, 1989), legislative committees (Gilligan \& Krehbiel, 1990) and political correctness (Morris, 2001) to double auctions (Matthews \& Postlewaite (1989); Farrell \& Gibbons (1989)), stock recommendations (Morgan \& Stocken, 2003) and matching markets (Coles, Kushnir, \& Niederle, Forthcoming).

${ }^{2}$ The list includes neologism proofness (Farrell, 1993), announcement proofness (Matthews, Okuno-Fujiwara \& Postlewaite, 1991), Partial Common Interest (Blume, Kim \& Sobel, 1993), the recurrent mop (Rabin \& Sobel, 1996) and No Incentive To Separate (Chen, Kartik \& Sobel, 2008)

${ }^{3}$ See Blume, DeJong, Kim \& Sprinkle (1998) and Agranov \& Schotter (2011) for studies on the role of language in cheap talk games.
} 
ACDC assumes that credible deviations matter for the stability of equilibria but that they matter in a gradual manner. On this basis, ACDC can select most plausible equilibria (ACDC equilibria), even in games where no equilibrium is entirely stable.

In this study, we test the assumption of ACDC that credible deviations matter gradually. In addition, to determine its value added more sharply, we test the predictions of ACDC in games where existing criteria are not predictive. We use the class of continuous external veto threat games introduced in De Groot Ruiz, Offerman \& Onderstal (2012b). These games allow for a clean manipulation of the size and frequency of credible deviations. Furthermore, they can have a large equilibrium set, which previous concepts cannot refine.

Our experimental design consists of five veto threat games and allows us to test four hypotheses. First, we test whether credible deviations matter at all: do neologism proofness and announcement proofness have any bite indeed? Second, we test whether the ACDC equilibrium performs best if all equilibria are unstable according to neologism proofness and announcement proofness. Third, we look at whether the ACDC equilibrium in similar games performs worse when its stability according to ACDC decreases. Finally, we test whether ACDC can explain behavior in case there is a large set of equilibria. The experimental results are supportive of all four hypotheses. This provides evidence that ACDC is able to predict well.

In order to better explain the dynamics of our data, we introduce a 'neologism dynamic.' The neologism dynamic is a simple best response dynamic with the additional feature that Senders send credible neologisms, which are also believed by Receivers. In contrast to a best response dynamic and a level- $k$ analysis, the neologism dynamic is predictive. In particular, the neologism dynamic supports the conclusions of ACDC and can explain the main dynamic characteristics of the data.

Turning to the literature, we see that relatively little experimental work exists on equilibrium selection in cheap talk games. Blume, DeJong \& Sprinkle (2001) test the predictions of the Partial Common Interest (PCI) criterion in a series of 
discrete games and compare it with neologism proofness. ${ }^{4}$ ACDC can explain Blume et al.'s experimental data at least as well as PCI (see De Groot Ruiz, Offerman \& Onderstal (2012a)). Experimental work on the Crawford-Sobel uniform quadratic game provides evidence that the most informative equilibrium performs best (Dickhaut, McCabe \& Mukherji (1995), Cai \& Wang (2006) and Wang, Spezio \& Camerer (2010)). ${ }^{5}$ This is predicted by the No Incentive To Separate (NITS) criterion (Chen, Kartik \& Sobel, 2008) as well as ACDC. Furthermore, it shows that as the bias parameter becomes smaller, the most informative equilibrium performs better, which is also predicted by ACDC. The present study is the first systematic experimental test of whether and to what extent credible deviations matter for the stability of cheap talk equilibria. In addition, it presents a rigorous test of ACDC in new experiments. ${ }^{6}$

This paper has the following structure. In section 2, we discuss the theory we require to derive the hypotheses we wish to test in our experiment. We present the experimental games we study, introduce ACDC and discuss the issue of equilibrium selection. In section 3 , we provide the experimental design. In section 4 we present the experimental results in relation to equilibrium selection. In section 5, we look at the dynamic aspects of our data and discuss the neologism dynamic. In section 6 , we introduce and discuss two additional treatments to test the robustness of our results. Section 7 concludes. Proofs are relegated to Appendix A.

\footnotetext{
${ }^{4}$ A partition of the typeset satisfies PCI "if types in each partition element unambiguously prefer to be identified as members of that element, and there is no finer partition with that property." The PCI criterion predicts that types in different elements send different messages.

${ }^{5}$ The focus of our paper is on what makes communication of private information credible. A different strand of the experimental literature deals with the question how players can credibly communicate their intentions (Davis \& Holt (1988), Cason (1995), Charness (2000), Ellingsen \& Johannesson (2004), Gneezy (2005), Charness \& Dufwenberg (2006), Lundquist, Ellingsen, Gribbe \& Johannesson (2009), Serra-Garcia, Van Damme \& Potters (2011)). Crawford (1998) provides a survey of papers on cheap talk experiments and DellaVigna \& Gentzkow (2010) review field evidence on persuasive communication.

${ }^{6}$ The model we present is a cheap talk bargaining game with asymmetric information. Experimental work on bargaining games with private information includes Radner \& Schotter (1989), Forsythe, Kennan \& Sopher (1991), Rapoport \& Fuller (1998), Rapoport, Erev \& Zwick (1995), Daniel, Seale \& Rapoport 1998), Valley, Moag \& Bazerman (1998), Schotter, Snyder \& Zheng (2000) and Croson, Boles \& Murnighan (2003).
} 


\section{Theory}

In this section, we develop the theory we use to construct the hypotheses for our experiment. In subsection 2.1 we introduce the game $\mathrm{G}(B)$, on which our main treatments are based, and apply existing refinements to this game. In 2.2, we introduce ACDC and show how it works out in $\mathrm{G}(B)$.

\section{1. $\mathrm{G}(B)$}

$\mathrm{G}(B)$ is a two-player veto threats cheap talk game between an informed Sender and an uninformed Receiver. ${ }^{7}$ The outcome of the game $x$ is a point in the interval $[0, B]$ or the disagreement point $\delta \notin \mathbb{R}$, where $B$ stands for boundary. The payoffs of the Receiver and the Sender are given by $U^{R}(x)$ and $U^{S}(x, t)$ :

$$
\begin{aligned}
& U^{R}(x)=60-\frac{2}{5} x \text { for all } x \in[0, B] \\
& U^{S}(x, t)=60-|x-t| \text { for all } x \in[0, B] \\
& U^{R}(\delta)=U^{S}(\delta, t)=0
\end{aligned}
$$

On the interval, the Receiver prefers smaller outcomes to larger outcomes. The payoffs of the Sender on the interval depend on her type $t$, which is drawn from a uniform distribution on $[0, B]$. The larger the distance between $t$ and $x \in[0, B]$, the lower the Sender's payoff. Both players receive a payoff of 0 if $\delta$ is the outcome, regardless of $t$. The Receiver prefers all outcomes on the line smaller than 150 to $\delta$; the Sender prefers $\delta$ to all outcomes on the line more than 60 away from her type $t$.

At the start of the game, nature draws a type $t$. Everything is common knowledge, except $t$. The game then proceeds as follows. Nature informs the

\footnotetext{
${ }^{7}$ We will refer to the Sender as a 'she' and the Receiver as 'he.'
} 
Sender of $t$. Subsequently, the Sender sends a costless message $m$ to the Receiver. Next, the Receiver proposes action $a \in[0, B]$. Finally, the Sender accepts $(v$ $=1)$ or rejects $(v=0)$. If the Sender accepts, $a$ is the outcome of the game, and if she rejects, $\delta$ is the outcome. Note that all messages are costless for the Sender. We assume $B \geq 120$ because under this condition the boundary does not affect the set of equilibrium actions. The model is close to the cheap talk game with veto threats of Matthews (1989). The main difference is that in our model the disagreement point does not lie on the interval.

A strategy for the Sender consists of a message strategy $\mu: T \rightarrow M$ and an acceptance strategy $\nu: A \times T \rightarrow\{0,1\}$. The strategy of the Receiver is an action strategy $\alpha: M^{\mathrm{TM}} A .^{8}$ Let $\Sigma^{S}$ be the set of Sender strategies and $\Sigma^{R}$ the set of Receiver strategies. Let $\{\mu, \alpha, \nu\}$ be a strategy profile and $\Sigma$ the set of all strategy profiles. Define $V^{R}(x, t ; \nu)=U^{R}(x, t) \cdot \nu(x, t)+U^{R}(\delta, t) \cdot(1-\nu(x, t))$ and $V^{S}(x, t ; \nu)=U^{S}(x, t) \cdot \nu(x, t)+U^{S}(\delta, t) \cdot(1-\nu(x, t))$. Finally, let the Receiver have prior beliefs $\beta^{0}(t)=f(t)$ and posterior beliefs $\beta(t \mid m)$ respectively. A pure strategy perfect Bayesian equilibrium (equilibrium henceforth) $\sigma=\{\mu, \alpha, \beta\}$ is characterized by the following four conditions:

For each $t \in T, m(t) \in \arg \max V^{S}(\alpha(m), t ; \nu)$

(2) For each $m \in M, \alpha(m) \in \arg \max _{a \in A} \int_{T} V^{R}(a, t ; \nu) \beta(t \mid m) d t$ $\nu(a, t)=1$ if $U^{S}(a, t)>U^{S}(\delta, t)$ and $\nu(a, t)=0$ if $U^{S}(a, t)<U^{S}(\delta, t)$ $\beta(m)$ is derived from $\mu$ and $\beta^{0}$ using Bayes Rule whenever possible

We say a type $t$ induces action $a$, if the Receiver always takes action $a$ after any message $t$ sends in equilibrium.

\footnotetext{
${ }^{8}$ For ease of exposition, we define equilibrium for pure strategies. For our purposes this is not restrictive. The refinement we will use requires Receivers to play pure strategies. In addition, all equilibrium outcomes are partition equilibria outcomes that can be supported by a pure Sender strategy.
} 
We consider the following type of equilibria. Following Matthews, we require the Receiver to play pure strategies and require Senders to always induce actions that are payoff-maximizing (in the spirit of Selten's (1975) trembling hand perfection). From now on, we will use 'equilibrium' to refer to a perfect Bayesian equilibrium satisfying these two requirements. ${ }^{9}$ As we show in De Groot Ruiz, Offerman \& Onderstal (2012b), all equilibria in such veto threat games are partition equilibria. A partition equilibrium can be characterized by the finite set of actions $A^{*}=\left\{a_{1}, a_{2}, \ldots, a_{n}\right\}$ the Receiver proposes in equilibrium, where $a_{1}<a_{2}<\ldots<a_{n}$. The number of equilibrium actions $n$ is called the size of the equilibrium. We say a type $t$ induces an action $a^{\prime}$, and write $a(t)=a^{\prime}$, if the Receiver proposes $a^{\prime}$ after any message $m$ the Sender sends. Each type induces an action $a_{i} \in A^{*}$ which maximizes her payoff and accepts it if and only if $U^{S}\left(a_{i}, t\right) \geq 0 .{ }^{10}$ (In $\mathrm{G}(B)$ the payoff maximizing action is simply the action closest to her type.) This means that a partition of the type space $0=t_{0}<t_{1}<\cdots<t_{n-1}<t_{n}=1$ exists such that each type in $\left(t_{i-1}, t_{i}\right)$ induces $a_{i}$.

It is straightforward to check that a set of actions $a_{1}<a_{2}<\ldots<a_{n}$ characterizes a partition equilibrium if and only if

$$
\begin{aligned}
& a_{i} \in \arg \max _{a \in A} \int_{t_{i-1}}^{t_{i}} U^{R}(a) I_{\left\{\tau: U^{S}(a, \tau) \geq 0\right\}}(\tau) d \tau \text { for all } i=1, \ldots, n, \text { where } \\
& t_{0}=0, t_{n}=1 \text { and } U^{S}\left(a_{i}, t_{i}\right)=U^{S}\left(a_{i+1}, t_{i}\right) \text { for all } i=1, \ldots, n-1 .
\end{aligned}
$$

The game has two equilibria: a pooling (size-1) equilibrium and a (semi)separating size-2 equilibrium. For both equilibria, the set of equilibrium actions the Receiver takes does not depend on $B$ :

\footnotetext{
${ }^{9}$ There is an infinite number of equilibria that induce the same equilibrium outcome. These essentially equivalent equilibria just differ in the messages that are used. For simplicity, we refer to a class of equilibria inducing the same equilibrium outcome simply as 'an equilibrium.'

${ }^{10}$ There will be a set of measure zero of types for which $U^{S}\left(a_{i}, t\right)=U^{S}\left(a_{i+1}, t\right)$ for some $i$. It does not matter which action they induce.
} 
Proposition $1 \mathrm{G}(B)$ has two equilibria: a pooling equilibrium $\sigma^{P}\left\{a_{1}=45\right\}$ and a separating equilibrium $\sigma^{S} \quad\left\{a_{1}=0, a_{2}=60\right\}$.

In the pooling equilibrium, all types induce 45, whereas in the separating equilibrium all Sender types in the interval $[0,30)$ induce action $a=0$ and all Sender types in the interval $(30, B]$ induce action $a=60$. In the separating equilibrium, the Sender always accepts, and in the pooling equilibrium all Senders in $[0,105]$ accept.

The intuition behind the proposition is the following. Since all equilibria are partition equilibria, the Receiver's posterior beliefs consist of intervals. If the Receiver believes the Sender's type is uniformly distributed on an interval $[\underline{t}, \bar{t}]$, he faces the following trade-off when looking for a best response. As the proposed action increases (up to $\min \{\underline{t}+60, \bar{t}-60\}$ ), the probability of acceptance increases but the utility conditional on acceptance decreases. Senders best respond by inducing the action closest to their type. As in any cheap talk game, there is a pooling equilibrium in which all Senders employ the same message strategy and the Receiver ignores all messages. In $\mathrm{G}(B)$, also a size-2 equilibrium exists. Higher size equilibria do not exist, roughly because there is a minimum distance between two positive equilibrium actions and a maximum to the value an equilibrium action can take.

At this point two questions arise. First, is one equilibrium more plausible than the other? Second, does $B$ influence the stability of the equilibria? Standard signaling refinements in the vein of Kohlberg and Mertens' (1986) strategic stability have no bite in cheap talk games since messages are costless. For a similar reason, the (Agent) Quantal Response Equilibrium (McKelvey \& Palfrey, 1998), which can often select equilibria in signaling games, is not predictive in cheap talk games. ${ }^{11}$ To address the selection problem for costless communication, Farrell (1993) considered credible deviations from equilibrium. He argued

\footnotetext{
${ }^{11}$ The Agent Quantal Response Equilibrium (A-QRE) is the extensive form game variant of the Quantal Response Equilibrium. The pooling equilibrium of a Cheap Talk game is always a limiting principal branch A-QRE. We come back to A-QRE in section 5.3.
} 
that, in contrast to the standard assumption in game theory, communication proceeds through a pre-existing natural language. Hence, people can use 'neologisms,' out-of-equilibrium messages with a literal meaning that will be understood (although not necessarily believed). Farrell considers neologisms which literally say: "propose action $\tilde{a}$, because my type is in set $N$." Farrell deems a neologism credible if and only if $(i)$ all types $t$ in $N$ prefer $\tilde{a}$ to their equilibrium action $a(t),(i i)$ all types $t$ not in $N$ prefer their equilibrium action $a(t)$ to $\tilde{a}$ and (iii) the best response of the Receiver after restricting the support of his prior to $N$ is to play $\tilde{a} \cdot{ }^{12}$ We will denote credible neologisms by $\langle\tilde{a}, N\rangle$. An equilibrium is neologism proof if it does not admit any credible neologism. Farrell argues that only neologism proof equilibria are stable, since rational players would move away from equilibria which admit credible neologisms.

Matthews, Okuno-Fujiwara \& Postlewaite (1991) consider more elaborate messages, called announcements, and propose three types of credible deviations. Weakly credible announcements are similar to neologisms, but allow deviating types to distinguish amongst themselves. A weakly credible announcement that should be believed if the Receiver realizes that types can send multiple announcements is a credible announcement. A credible announcement that survives a rigorous Stiglitz-critique is strongly credible. Equilibria that admit no (weakly/strongly) credible announcements are called (strongly/weakly) announcement proof. In $\mathrm{G}(B)$, strong announcement proofness, announcement proofness and neologism proofness coincide. ${ }^{13,14}$ Consequently, for ease of exposition we can limit our discussion of credible deviations to credible neologisms. ${ }^{15}$

In $\mathrm{G}(B)$ two types of credible neologisms can exist. A 'low' neologism which roughly says "I am a low type and prefer 0 to the lowest equilibrium action and

\footnotetext{
${ }^{12}$ Farrell does not consider cheap talk games with veto threats. In line with the trembling hand refinement, we assume that types induce a neologism if $U^{S}(\tilde{a}, t)>U^{S}(a(t), t)$.

${ }^{13}$ The reasons are that all weakly credible announcements are equivalent to a credible neologism (for similar arguments that there is at most a size-2 equilibrium) and that all types can send at most one credible neologism.

${ }_{14}$ All equilibria in $\mathrm{G}(B)$ are weakly announcement proof, as in almost all cheap talk games.

${ }^{15}$ Myerson (1989) introduces credible negotiation statements, which are similar to credible neologisms. Myerson is able to obtain a solution concept that guarantees existence but at the cost of assuming the presence of a mediator.
} 
so do you, so play 0" and a 'high' neologism which roughly says "I am a high type, and it is probable that I will not accept the highest equilibrium action, so it is better for both of us if you propose something higher." As the following proposition shows, the pooling equilibrium is never neologism proof and the separating equilibrium is only neologism proof if $B=120$.

Proposition $\mathbf{2}$ The pooling equilibrium admits the credible neologisms $\langle 0,[0,22.5]\rangle$ and $\left\langle\min \{B-60,75\},\left[\min \left\{\frac{B-15}{2}, 60\right\}, B\right]\right\rangle$. The separating equilibrium is neologism proof if $B=120$. For $B>120$, the separating equilibrium admits the credible neologism $\left\langle\min \{B-60,80\},\left[\min \left\{\frac{B}{2}, 70\right\}, B\right]\right\rangle$.

So, for $B>120$ neologism proofness (and announcement proofness) are silent about the stability of the separating equilibrium relative to the pooling equilibrium. In addition, they are silent about whether the separating equilibrium is more stable if $B=121$ than, say, if $B=210$. In De Groot Ruiz, Offerman \& Onderstal (2012a) we show that the same holds for other cheap talk refinements including communication proofness (Blume \& Sobel, 1995) and the recurrent mop (Rabin \& Sobel, 1996), as well as the non-equilibrium concepts of Credible Message Rationalizability (Rabin, 1990) and PCI (Blume, Kim \& Sobel, 1993).

The NITS (Chen, Kartik \& Sobel, 2008) criterion, which successfully predicts in the Crawford-Sobel (1982) game, is partially predictive in $\mathrm{G}(B)$. NITS starts by specifying a 'lowest type,' a type with the property that all other types prefer to be revealed as themselves rather than as that lowest type. An equilibrium survives NITS if the lowest type has no incentive to separate, i.e. if the lowest type prefers her equilibrium outcome to the outcome she would get if she could reveal her type. In our game, such a 'lowest type' cannot easily be formulated. If we take $t=0$ as the lowest type, for each $B$ only the separating equilibrium is 
NITS in our game. ${ }^{16}$ Hence, NITS would predict that the separating equilibrium is always stable regardless of $B$. (In section 6.2, we introduce a game that has multiple equilibria that survive NITS.)

Finally, some may argue that the most influential equilibrium (i.e. the equilibrium which induces the largest number of actions) is the most plausible equilibrium, aside of any stability considerations. In our game, this criterion also selects the separating equilibrium regardless of $B$.

In sum, existing criteria provide no or a partial answer to the question how stable equilibria in $\mathrm{G}(B)$ are for $B>120$.

\section{2. $\mathrm{ACDC}$ in $\mathrm{G}(B)$}

In our view, the idea of credible deviations is sound, but a rationalistic binary division between stable and unstable equilibria is inadequate to fully capture the intricate patterns of human behavior. Our conjecture is that two aspects will affect the behavioral stability of an equilibrium. The first concerns the mass of types that can credibly induce a deviation. The smaller this mass becomes, the less unstable an equilibrium will be, as it will be disturbed less frequently. The second aspect concerns how much the deviation profile differs from the equilibrium profile in terms of Sender payoffs. The smaller this difference becomes, the smaller both the Sender's incentive to deviate and the perturbation to the equilibrium if she deviates will be.

For instance, the separating equilibrium is not neologism proof if $B=121$. However, we do not expect behavior in the game $\mathrm{G}(121)$ to be very different to behavior in $G(120)$. After all, the induced deviations from equilibrium are very small: types in $[60.5,121]$ induce 61 instead of 60 . Hence, Senders can at most earn 1 by deviating and, if they deviate, the resulting profile is very similar to the equilibrium profile. In contrast, in the pooling equilibrium the neologism

\footnotetext{
${ }^{16}$ All types in $[0,60]$ are lowest types according to Chen et al.'s definition. The pooling equilibrium survives NITS relative to types in $[22.5,105]$, whereas the separating equilibrium survives NITS relative to types in $[0,30]$.
} 
deviations are substantial: types from 0 to 22.5 deviate from 45 to 0 , and types from 53 to 121 deviate from 45 to 61 . As a consequence, Senders have a large incentive to deviate to a profile which is very different from the equilibrium profile. Furthermore, the separating equilibrium seems more stable if $B=121$ than if, say, $B=210$, when types in $[70,210]$ can credibly induce 80 rather than 60.

In De Groot Ruiz, Offerman \& Onderstal (2012a), we formalize these ideas in the concept of the Average Credible Deviation (ACD). ${ }^{17}$ The ACD intends to capture the frequency and intensity of deviations. We need a couple of definitions. Let $a^{\sigma}(t)$ be the equilibrium action induced by type $t$ in equilibrium $\sigma$; and let $\tilde{a}^{\sigma}(t)$ be the deviating action type $t$ induces if she plays a credible neologism. ${ }^{18}$ Let $\tilde{a}^{\sigma}(t)=a^{\sigma}(t)$ if Sender type $t$ cannot play a credible neologism. Finally, we define $\underline{U}^{S}(t)$ and $\bar{U}^{S}(t)$ as the lowest and highest payoff a Sender can get if both players play a rationalizable strategy. Now, for each Sender type $t$, we specify the size of the credible deviation from equilibrium, $C D(t, \sigma)$. The ACD is the expected value of the credible deviations. We measure the size of a credible deviation by the Sender's incentive to deviate relative to the largest (rationalizable) incentive possible, so that it lies on a scale between 0 and 1 . The higher this incentive is, the higher the probability that a Sender will deviate and the larger the upheaval such a deviation can cause to an equilibrium. In particular, we define the credible deviation for type $t$ as

(4)

$$
C D(t, \sigma) \equiv \frac{U^{S}\left(t, \bar{a}^{\sigma}(t)\right)-U^{S}\left(t, a^{\sigma}(t)\right)}{\bar{U}^{S}(t)-\underline{U}^{S}(t)}
$$

\footnotetext{
${ }^{17}$ There we also provided a more general and rigorous treatment of the concept. Here we restrict ourselves to the definitions needed in the current setting.

${ }^{18}$ In $\mathrm{G}(B)$, ACDC gives equivalent results if we use weakly or ordinary credible announcements are used instead of credible neologisms, as is the case in many games. For cases where the theories differ, we prefer (ordinary) credible announcements for discrete games and credible neologisms for continuous games.
} 
whenever $U^{S}\left(t, a^{\sigma}(t)\right)>\underline{U}^{S}(t)$. If $U^{S}\left(t, a^{\sigma}(t)\right)=\underline{U}^{S}(t)$, the Sender has no incentive to adhere to her equilibrium strategy, as she can do no worse by deviating, and we set $C D(t, \sigma) \equiv 1$. The ACD of equilibrium $\sigma$ is now defined as

(5) $A C D(\sigma)=E_{t}[C D(t, \sigma)]$

Observe that $A C D(\sigma) \in[0,1]$. Using $\mathrm{ACD}$, we can formulate the ACD-Criterion (ACDC). ACDC predicts that an equilibrium $\sigma$ is more stable than an equilibrium $\sigma^{\prime}$ if $A C D(\sigma)<A C D\left(\sigma^{\prime}\right)$. Hence, ACDC allows us to select equilibria. In particular, we call an equilibrium an ACDC equilibrium if there is no other equilibrium in the game with a lower ACD. We consider an ACDC equilibrium as the most plausible equilibrium, i.e. that which will predict best on average, rather than the equilibrium that will always be played all of the time. ACDC can select equilibria when neologism proofness is silent and reduces to the latter if neologism proof equilibria exist.

The following proposition gives the results of ACDC for $\mathrm{G}(B)$.

Proposition 3 The separating equilibrium $\sigma^{S}$ is the unique ACDC equilibrium. Furthermore, the ACD of the separating equilibrium is 0 for $B=120$ (in which case the equilibrium is neologism proof) and strictly increasing in $B$.

We can now see why $G(B)$ provides a good testing ground for our ideas. It contains the features that make (continuous) cheap talk games difficult to refine. In contrast to Crawford \& Sobel's (1982) and Matthews' (1989) cheap talk models, however, in our game a parameter value exists such that there are multiple equilibria, of which only one is neologism proof. Hence, our model allows us to test the relevance of credible deviations in a continuous setting. Furthermore, it allows us to test the idea that stability is not all-or-nothing. First, we can compare within a game two equilibria that are not neologism 
proof. Second, across games we can gradually increase the number of types that can credibly deviate in an equilibrium by increasing $B$.

\section{Experimental Design and Procedures}

We ran five treatments. In three treatments, we ran $\mathrm{G}(B)$ with an increasing boundary: $\mathrm{G}(120), \mathrm{G}(130)$ and $\mathrm{G}(210)$. In addition we ran two additional robustness treatments $\mathrm{T} 4$ and $\mathrm{T} 5$ to which we return in section 6 . Table 1 summarizes the theoretical properties of the experimental treatments. For each treatment, we have six matching groups, each consisting of 10 subjects (5 Senders and 5 Receivers).

TABLE 1

SUMMARY OF EXPERIMENTAL DESIGN

\begin{tabular}{cccccc}
\hline \hline Treatment & $U^{R}(\delta)$ & $U^{S}(\delta)$ & $B$ & Equilibria $^{1}$ & $\mathrm{ACD}^{2}$ \\
\hline $\mathrm{G}(120)$ & 0 & 0 & 120 & $\{45\},\{0,60\}^{* *}$ & 0 \\
$\mathrm{G}(130)$ & 0 & 0 & 130 & $\{45\},\{0,60\}^{*}$ & 0.22 \\
$\mathrm{G}(210)$ & 0 & 0 & 210 & $\{45\},\{0,60\}^{*}$ & 0.50 \\
T4 & 30 & 30 & 120 & $\{45 / 2\},\{0,30\}^{*}$ & 0.56 \\
T5 & 0 & 30 & 120 & $\{30\},\left\{a_{1}, a_{1}+60\right\}$, & 0 \\
& & & $\left\{0, a_{2}, a_{2}+60\right\}^{* * 3,4}$ & \\
\hline \hline
\end{tabular}

Notes: In each game, $\mathrm{t}$ was uniformly distributed on the integers in $[0, B] . U^{R}(x)=60-\frac{2}{3} x$ and $U^{S}(x, t)=60-|t-x| \cdot{ }^{1}$ An equilibrium has a ${ }^{*}$ if it is ACDC and $* *$ if it is neologism proof as well. ${ }^{2}$ The ACD of the ACDC equilibrium. ${ }^{3} a_{1} \in[0,30]$ and $a_{2} \in(0,30] .{ }^{4}$ Only $\{0,30,60\}$ is ACDC.

We used a standard procedure to recruit subjects from the student population of the University of Amsterdam. The computerized experiment was run at the CREED lab. The software was written with z-Tree (Fischbacher, 2007).

At the start of the experiment, subjects were randomly assigned to the role of Sender ('chooser' in the terminology of the experiment) or Receiver ('proposer'). Subjects kept the same role throughout the whole experiment. Subjects read the role-specific instructions on paper at their own pace. (See Appendix $\mathrm{C}$ for the instructions.) After reading the instructions, subjects had to answer several 
questions testing their understanding of the instructions. Only when all subjects had answered all questions correctly, the experiment started.

Subjects received a starting capital of 100 points. In addition, subjects earned points with their decisions in each of the 50 periods. (Subjects were informed that the experiment would last for approximately 50 periods.) At the end of the experiment, total point earnings were exchanged to euros at a rate of 1.5 euros for 100 points. In a session, we ran 2 matching groups simultaneously, each consisting of 5 Senders and 5 Receivers. In every period, each Sender was randomly rematched with a Receiver in the own matching group. In total, 300 subjects participated, who on average earned 28.30 euros in approximately 2 hours, with a minimum of 10.10 euros and a maximum of 40.47 euros. Each subject only participated once.

The procedure within a period was as follows. In each period, the Sender was informed of her own type. All subjects knew that each individual Sender's type in each period was an independent draw from the uniform distribution on $[0, B]$. ${ }^{19}$ After having been informed of the own type, each Sender sent a message ('suggestion' in the terminology of the experiment) to the Receiver. The Receiver was informed of the message but not of the Sender's type. Then the Receiver chose an action ('made a proposal') that was either accepted or rejected by the Sender. Types, messages and actions were confined to integers in $[0, B]{ }^{20}$

Payoffs were then calculated according to the payoffs in Table 1. At the end of the period, Senders and Receivers were informed of the state of the world (the Sender type) and all the decisions made by the pair they were part of. In addition, each subject was shown her own payoff and how it was calculated. At any moment, subjects were provided with information about the social history

\footnotetext{
${ }^{19}$ To maximize the comparability of the treatments, we drew three sets of types for one treatment and then rescaled these sets for each of the other treatments.

${ }^{20}$ We chose for this restricted message space instead of a free chat in order to be able to provide a history screen, facilitate learning and have data that can be interpreted clearly. Notice that the message space is rich enough for the communication of all credible neologisms in both equilibria, as in our game a neologism action uniquely identifies a credible neologism.
} 
in order to facilitate learning. ${ }^{21}$ At the bottom of the screen they saw how play had unfolded in the 15 most recent periods in their own matching group. For Senders the information was organized as follows. The left-hand side showed a table summarizing the choices of the pairs in the own matching group. Each row contained a pair's suggestion (message), proposal, acceptance and preferred outcome (type of the Sender). The table was first sorted on suggestion, then on proposal, acceptance and finally on preferred outcome. The right-hand side showed the corresponding graph that listed the proposals as function of the suggestions. Figure 1 shows an example of the information that Senders received.

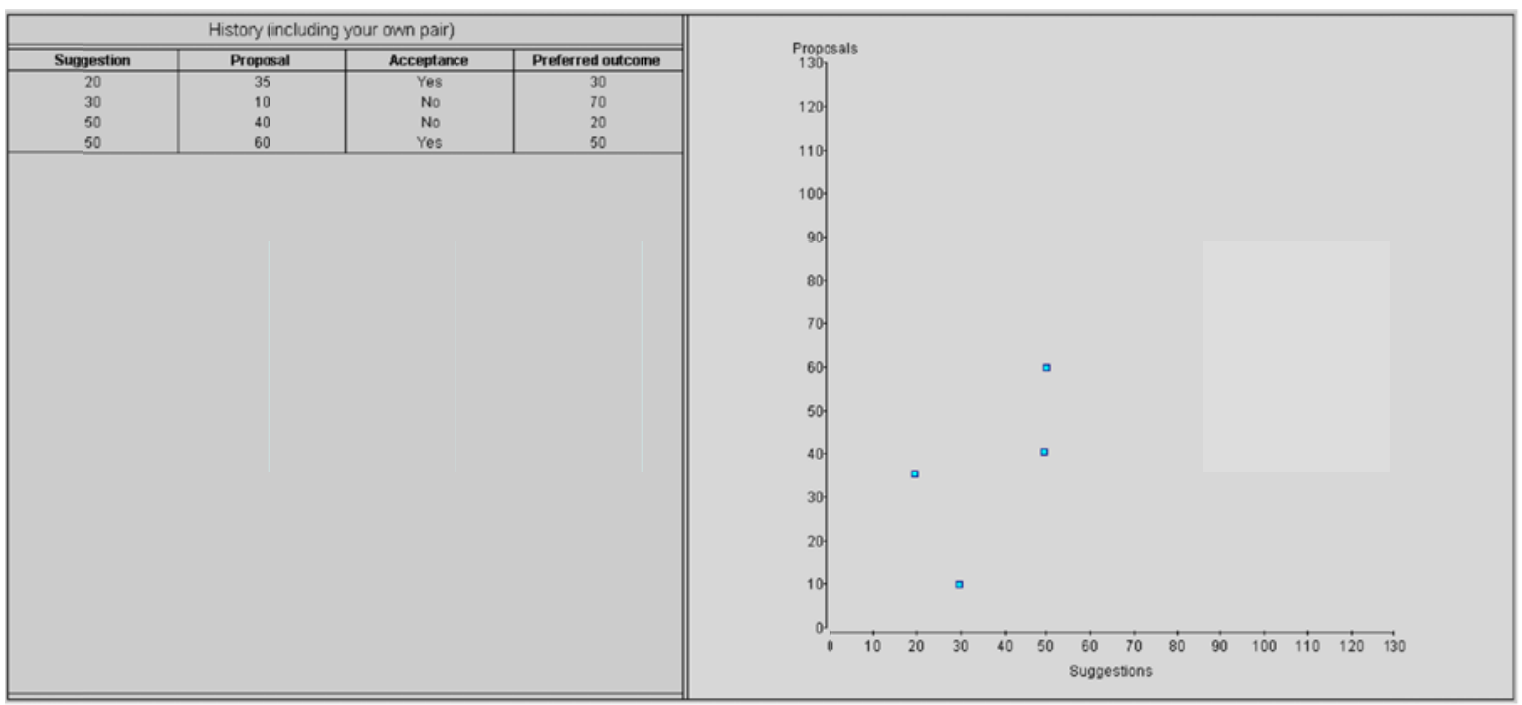

Figure 1

Example of social history screen History Screen (Senders)

For Receivers the information was communicated in a slightly different way. In their table, each row listed a pair's suggestion, preferred outcome, proposal and acceptance. The table was first sorted on message, then on preferred outcome, proposal and finally on acceptance. In their graph, preferred outcomes were shown as function of the suggestions.

\footnotetext{
${ }^{21}$ Miller \& Plott (1985) showed how a social history can help subjects understand the strategic nature of signaling games.
} 


\section{Experimental Results: Equilibrium Performance}

In this section, we discuss our experimental results in the light of equilibrium selection and performance. Since there are an infinite number of strategy profiles supporting the same outcome, we focus in our analysis mainly on the outcome, i.e. the mapping of types to actions and acceptance decisions. After describing the data, we provide statistics on acceptance rates, which shows that Senders accept proposals with a positive payoff according to equilibrium in the vast majority $(96 \%)$ of cases. Next, we deal with identifying which equilibrium is selected and how well each equilibrium performs. For this, we look at two performance measures: the percentage of outcomes an equilibrium predicts correctly (within some bandwidth) and the average prediction error in terms of the distance between predicted and actual proposals. On the basis of this we get a continuous rather than binary measure of equilibrium performance, in line with the methodological stance of ACDC that behavior is not fully in or out of equilibrium. On the basis of this performance measure, we can select which equilibrium performs best in relative terms.

As we are interested on equilibrium selection here, we focus exclusively on the final 15 periods of the experiment. In section 5 , we will take a closer look at the dynamics in the data and also use the data from early periods. All statistical tests have been done treating each matching group as one independent data point. For comparisons within a treatment we use Wilcoxon signed rank tests, and for comparisons between treatments we use Mann-Whitney ranksum tests. $*$, **, *** indicate significance at the $10 \%, 5 \%$ and $1 \%$ level respectively (for two-tailed tests).

We start with some descriptive statistics of the final 15 periods of the experiment. 

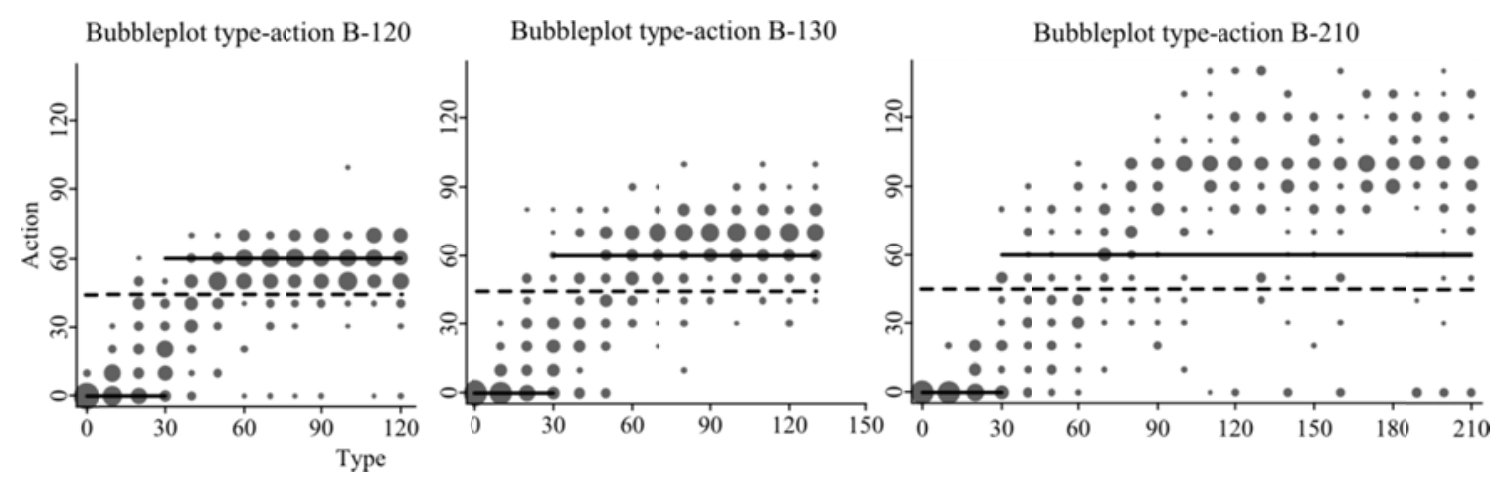

Figure 2

This figure shows what types (x-axis) induced what action (y-axis) for the last 15 periods. Bubbles are clustered on a 10-grid. Each bubble represents all observations in a $(t-5, t+5] \times(a-5, a+5]$ neighborhood. The size of each bubble is proportional to the number of observations it represents.

Figure 2 plots the Receivers' actions as a function of the Senders' types for each of the three treatments together with the equilibrium predictions. Consistent with the separating equilibrium we find that Senders with low types tend to elicit an action of 0 , while the Senders with high types tend to trigger a high action. Types close to the equilibrium indifferent type of 30 show a more continuous separation than the equilibrium step-function. 'High actions' are close to equilibrium for $B=120$ and, to a slightly lesser degree, for $B=130$. We define the high action as the action high types ( $t>30$, the equilibrium threshold) induce, conditional on being higher than the pooling action 45. In the G(120) treatment, where the separating equilibrium is neologism proof, the distribution of the high actions is similar and centered around 60. (The high actions are not significantly different from 60.) This is in agreement with the equilibrium prediction. We see a similar pattern in G(130), although the mean high action is now somewhat higher than the equilibrium action, as expected if high types send a neologism. In $G(210)$, the elicited actions by high types are far off from equilibrium and much more dispersed. (In both $\mathrm{G}(130)$ and $\mathrm{G}(210)$, the high action is significantly larger than 60.)

The equilibrium analysis assumes that Senders do not leave money on the table, i.e., they are supposed to accept any action that gives them positive payoff. Overall, Senders accepted proposed actions that would give them a positive payoff in $96 \%$ of the cases. Table 2 presents the actual acceptance 
frequencies as function of the Sender's payoff (in the rows) and the Sender's share in the total payoff (in the columns). It is remarkable that Senders almost always accept 'decent' proposals that give them at least 10, independent of their share in the total payoff. The share in total payoff only matters when Senders receive 'peanuts' proposals with payoffs below 10. As a result, the equilibrium assumption about Senders' acceptance behavior is by and large supported in the data. Notice that the picture about acceptance rates differs from results in standard ultimatum games, where subjects tend to reject proposed actions more often (Oosterbeek, Sloof \& Van der Kuilen, 2004). A crucial difference between our bargaining game and the ultimatum game is that in our game the Receiver is not informed of the type of the Sender. Therefore, unlike in the ultimatum game, it is unclear whether an unfavorable proposal is made intentionally.

TABLE 2

ACCEPTANCE RATES

\begin{tabular}{|c|c|c|c|c|c|c|c|c|c|c|}
\hline \multicolumn{11}{|c|}{ Share Sender } \\
\hline $\begin{array}{l}\text { Payoff } \\
\text { Sender }\end{array}$ & \multicolumn{2}{|c|}{ Total } & $0-10 \%$ & $10-20 \%$ & $20-30 \%$ & $30-40 \%$ & \multicolumn{2}{|c|}{$40-50 \%$} & \multicolumn{2}{|c|}{$>50 \%$} \\
\hline$<0$ & $0 \%$ & 211 & - & - & - & - & - & & - & \\
\hline$=0$ & $50 \%$ & 6 & $50 \% \quad 6$ & - & - & - & - & & - & \\
\hline $0-10$ & $70 \%$ & 112 & $40 \% \quad 30$ & $73 \% \quad 49$ & $90 \% \quad 29$ & $100 \%$ & $100 \%$ & 1 & $100 \%$ & 1 \\
\hline $10-20$ & $92 \%$ & 122 & - & $100 \% 2$ & $87 \% \quad 69$ & $97 \% \quad 36$ & $100 \%$ & 8 & $100 \%$ & 7 \\
\hline $20-30$ & $99 \%$ & 135 & - & - & $100 \% \quad 13$ & $100 \% \quad 48$ & $98 \%$ & 59 & $100 \%$ & 15 \\
\hline $30-40$ & $100 \%$ & 182 & - & - & - & $100 \% 28$ & $100 \%$ & 76 & $100 \%$ & 78 \\
\hline $40-50$ & $100 \%$ & 250 & - & - & - & - & $100 \%$ & 89 & $99 \%$ & 161 \\
\hline \multirow[t]{2}{*}{$50-60$} & $100 \%$ & 332 & - & - & - & - & $100 \%$ & 76 & $100 \%$ & 256 \\
\hline & $\begin{array}{r}\text { Total } \\
\text { payc }\end{array}$ & $\begin{array}{l}\text { when } \\
\text { off }>0\end{array}$ & $40 \% \quad 36$ & $75 \% \quad 51$ & $89 \% \quad 111$ & $99 \% \quad 114$ & $100 \%$ & 309 & $100 \%$ & 518 \\
\hline
\end{tabular}

Notes: the cells show the acceptance rates (in percentages) as a function of the surplus payoff the Sender would receive if she would accept the proposed action (in the rows) and her share of the surplus (in the columns, cases where the surplus was not positive were dropped). The number of observations pertaining to the cell is listed in italics. The Sender's share is defined as $100 \% \times \frac{U^{S}(a, t)}{U^{S}(a, t)+U^{R}(a)}$.

Table 3 reports how often actual play was close to equilibrium. We say that an outcome is consistent with equilibrium ('correctly predicted') if the actual action lies within a bandwidth of 10 and if the acceptance decision was correctly predicted. The absolute numbers in the table are obviously dependent on the chosen bandwidth. Here, we are interested in the relative magnitudes of the 
numbers, for which the exact level of the bandwidth turns out not to matter. In addition, we also look at the average (absolute) prediction error of the equilibria, reported in Figure 3. Let $a^{\sigma}(t)$ be the equilibrium action of the Receiver given type $t$ and $\hat{a}_{i}\left(t_{i}\right)$ the observed action for observation $i$. The average prediction error (for a set of $n$ observations $I$ ) is then $\frac{1}{n} \sum_{i \in I}\left|\hat{a}_{i}\left(t_{i}\right)-a^{\sigma}\left(t_{i}\right)\right|$. The percentage of outcomes that are correctly predicted is an intuitive measure of predictive success, whereas the average prediction error a parameter-free and precise measure. The results are qualitatively identical (and equally significant) for both measures of predictive success.

\section{TABLE 3}

Fraction of Outcomes Correctly Predicted By Equilibria

\begin{tabular}{ccccccc}
\hline \hline & \multicolumn{3}{c}{ All observations } & \multicolumn{3}{c}{ Observations with $t<120$} \\
\hline Treatment & \multicolumn{2}{c}{ Equilibria } & Dif & \multicolumn{2}{c}{ Equilibria } & Dif \\
& Pooling & Separating & & Pooling & Separating & \\
\hline G $(120)$ & .39 & .69 & $.30^{* *}$ & .39 & .69 & $.30^{* *}$ \\
G $(130)$ & .13 & .57 & $.43^{* *}$ & .14 & .59 & $.45^{* *}$ \\
G $(210)$ & .09 & .18 & $.09^{* *}$ & .09 & .28 & $.19^{* *}$ \\
Dif $120-130$ & $.25^{* *}$ & .12 & & $.25^{* *}$ & .09 & \\
Dif $120-210$ & $.30^{* * *}$ & $.51^{* * *}$ & & $.29^{* *}$ & $.40^{* * *}$ & \\
Dif $130-210$ & $.05^{* *}$ & $.39^{* * *}$ & & .05 & $.31^{* * *}$ & \\
\hline \hline
\end{tabular}

Notes: The table shows per treatment the median (over matching groups) of the fraction of correctly predicted outcomes by the equilibrium. We classified a prediction as correct if both $(i)$ the distance between the predicted and observed action was not larger than 10 and (ii) the acceptance decision was correctly predicted. We used the data of the last 15 periods. 'Dif' denotes 'difference.'

We first deal with the question whether credible deviations (and hence neologism proofness and announcement proofness) have a bite. For this question, two comparisons are relevant. First, within treatment G(120) the separating equilibrium is neologism proof while the pooling equilibrium is not. In accordance with neologism proofness, more outcomes are consistent with the separating equilibrium than with the pooling equilibrium. The difference is both substantial and statistically significant. Second, when we move from treatment G(120) to $\mathrm{G}(210)$, the separating equilibrium ceases to be neologism proof. While the separating equilibrium does a good job in $\mathrm{G}(120)$, it organizes only a bleak 
minority of the data in $\mathrm{G}(210)$. This conclusion is valid when we take the data for all types as well as when we condition on the outcomes with types less than 120. So also when we compare the behavioral stability of the same equilibrium across treatments, we find support for neologism proofness.

To investigate whether credible deviations matter gradually across games in the manner ACDC predicts, we compare G(120), G(130) and G(210). In G(130), the separating equilibrium is no longer neologism proof but the ACD measure remains rather small relative to $\mathrm{G}(210)$. So if ACDC makes sense, the results of $\mathrm{G}(130)$ should be closer to $\mathrm{G}(120)$ than to $\mathrm{G}(210)$. Table 3 confirms that this is indeed the case. Like in $\mathrm{G}(120)$, the separating equilibrium is much more successful than the pooling equilibrium. The separating equilibrium predicts behavior a bit less in $G(130)$ than in $G(120)$, but the difference is not significant. ${ }^{22}$ In contrast, the difference between $\mathrm{G}(130)$ and $\mathrm{G}(210)$ is much larger. In $\mathrm{G}(210)$, significantly fewer cases are consistent with the separating equilibrium than in $\mathrm{G}(130)$. So even though the separating equilibrium is not neologism proof in either treatment, it traces the data much better in $G(130)$ than in $G(210)$, as predicted by ACDC. Observe that in G(210), ACDC does not only predict that behavior is closer to the separating equilibrium than to the pooling equilibrium, but also that it will be rather unstable due to its high ACD.

Regarding equilibrium selection, observe that in all treatments the ACDC (separating) equilibrium predicts significantly better than the non-ACDC (pooling) equilibrium. This is also the case for $\mathrm{G}(130)$ and $\mathrm{G}(210)$, where neither equilibrium is neologism proof. Still, in $\mathrm{G}(210)$, the difference between the separating and the pooling equilibrium is much smaller than in the other treatments, in line with ACDC.

The power of ACDC is further illustrated in Figure 3. This figure lists the average prediction error of a particular equilibrium and treatment as a function of its ACD. In agreement with ACDC, the higher the ACD measure, the larger the average prediction error tends to be. Notice in particular that the average

\footnotetext{
${ }^{22}$ Since this result also holds when the analysis is restricted to observations with $t \leq 120$, this is not a measurement artifact due to a change in the interval of measurement.
} 
prediction error of the separating equilibrium only rises slightly when it just ceases to be neologism proof (relative to the differences with $\mathrm{G}(210)$ ).

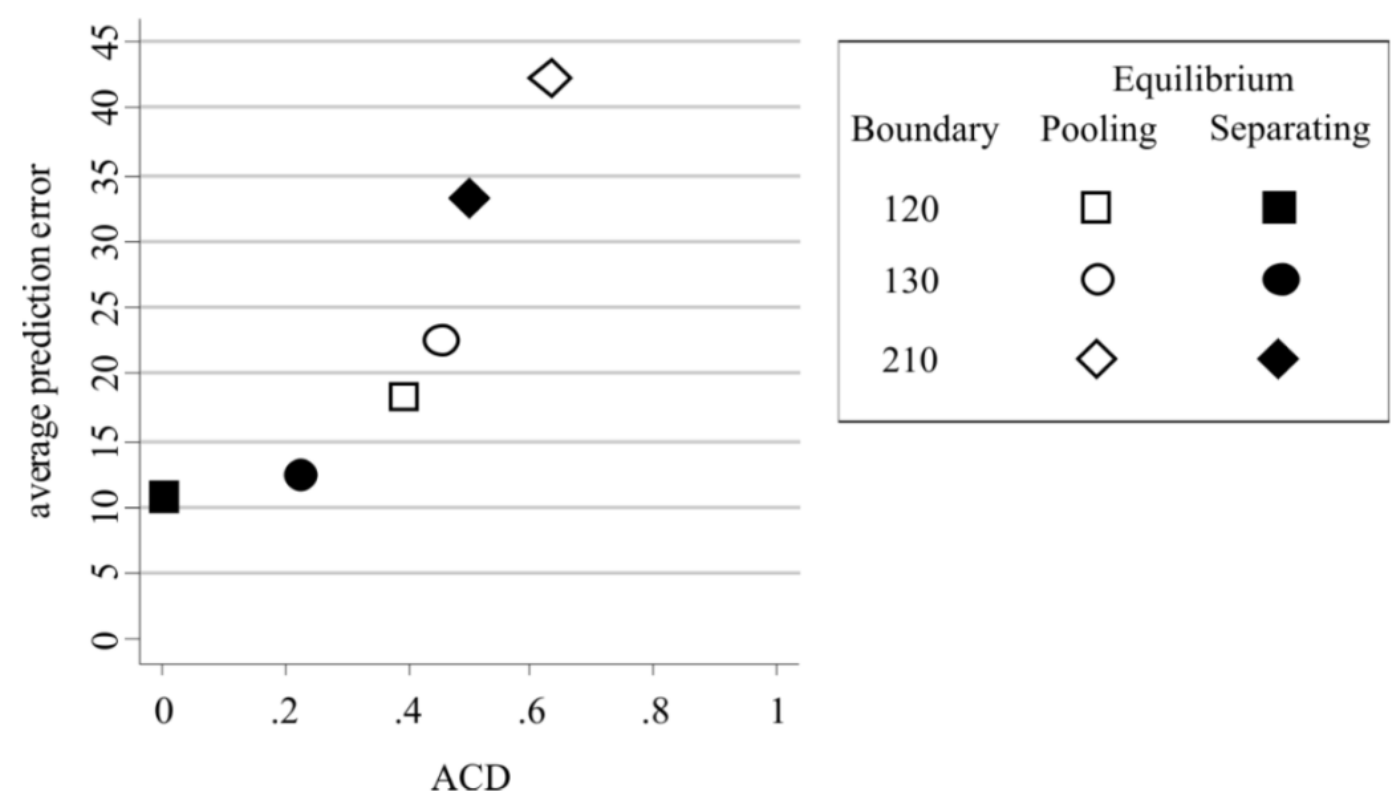

Figure 3

The figure plots for each equilibrium in each treatment its theoretical ACD against its empirical prediction error. We report the median (over matching groups) of the average prediction error. We used the data of the last 15 periods.

\section{Dynamics}

In this section, we look at the dynamics. In subsection 5.1, we describe the most important dynamic features of the data. In 5.2, we observe that an elementary best response model is not predictive. In 5.3, we introduce the neologism dynamic, which is able to explain important parts of the data.

\subsection{Dynamics in Experiment}

In this section we deal with the question how subjects adapted their behavior during the experiment. Figure 4 plots messages conditional on Sender type (lefthand side), actions conditional on message received (middle) and actions condi- 
tional on Sender type (right-hand side). We present plots for the first 15 and last 15 periods in each treatment. The type-message plots show that Senders' messages are higher than their types and that Senders learn gradually to exaggerate more. In the last 15 periods of each treatment, Senders overstate the true state more than in the first 15 periods. Thus, there is 'language inflation.'

Receivers' action-message plots provide the mirror image of Senders' typemessage plots. That is, in the first part of the experiment Receivers tend to propose actions slightly below the messages received. In the final part of the experiment, Receivers have learned to subtract larger amounts from the messages received. The type-action plots on the right hand side illustrate how close the actually triggered actions are to the equilibrium predictions. For treatments $\mathrm{G}(130)$ and $\mathrm{G}(120)$, the data are closer to the separating equilibrium in the final part of the experiment than in the first part of the experiment. A similar trend is not observed in $\mathrm{G}(210)$. To the contrary, in this treatment the data remain far from equilibrium throughout the whole experiment.

We now turn to the questions how easily subjects reached the separating equilibrium in the different treatments and how likely it was that they stayed there. Table 4 presents the relevant statistics separately for the first part (first 15 periods) and the final part (last 15 periods) of the experiment. In the first part of the experiment, subjects more easily reached the separating equilibrium from a state of disequilibrium in treatments $G(120)$ and $G(130)$ than in $G(210)$. When subjects were approximately playing according to the separating equilibrium in the previous period, they were much more likely to stay there in treatments $G(120)$ and $G(130)$ than in treatment $G(210)$. The lower part of the table shows that the differences between treatment $\mathrm{G}(210)$ and the other treatments became even more pronounced in the final part of the experiment. In particular, in $\mathrm{G}(120)$ and $\mathrm{G}(130)$ the separating equilibrium attracts more outcomes in the final part than in the first part (and in G(120) significantly so). In contrast, in $\mathrm{G}(210)$ the separating equilibrium attracts less outcomes in the final part; in fact, it hardly attracts any outcomes in the final part. 


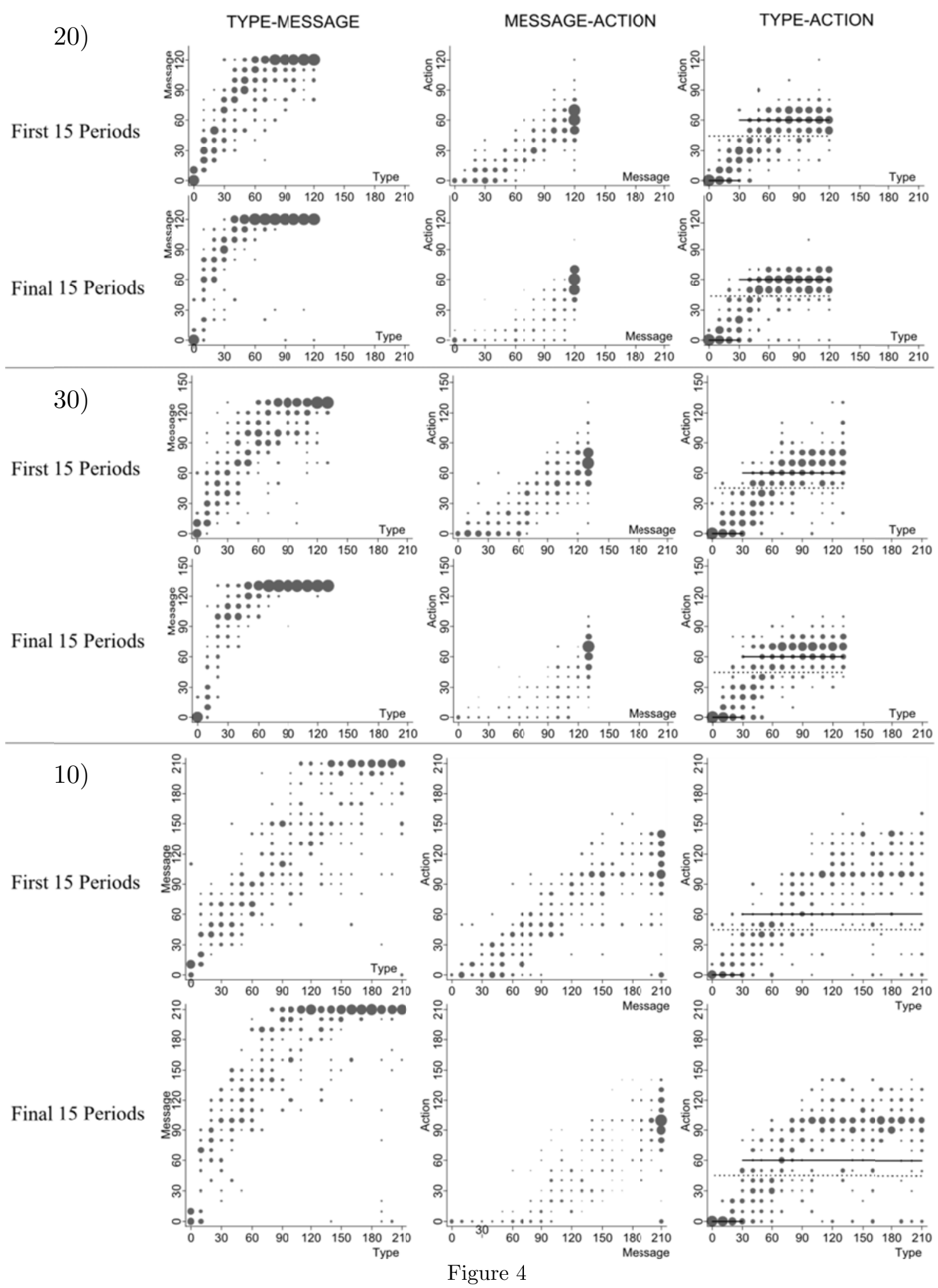

This figure compares the chosen strategies (type-message, message-action) and the resulting profile for the first 15 and last 15 rounds. The bubble plots are clustered on a grid of 10 . In the last column, the solid line represents the separating equilibrium and the dotted line the pooling equilibrium. 
TABLE 4

Fraction of Matching Group ObSERVATions in SEPARAting Equilibrium Conditional on Previous State

\begin{tabular}{cccc}
\hline \hline & Treatment & $\begin{array}{c}\text { Previous state } \\
\text { no equilibrium }\end{array}$ & $\begin{array}{c}\text { Previous state } \\
\text { equilibrium }\end{array}$ \\
\hline First Part & $\mathrm{G}(120)$ & .46 & .60 \\
(Periods 1-15) & $\mathrm{G}(130)$ & .41 & .51 \\
& $\mathrm{G}(210)$ & .10 & .09 \\
& Dif $120-130$ & .05 & .09 \\
& Dif $120-210$ & $.36^{* * *}$ & $.51^{* * *}$ \\
& Dif $130-210$ & $.31^{* * *}$ & $.42^{* * *}$ \\
\hline Final Part & $\mathrm{G}(120)$ & .64 & .74 \\
(Periods 36-50) & $\mathrm{G}(130)$ & .50 & .72 \\
& $\mathrm{G}(210)$ & .03 & .00 \\
& Dif $120-130$ & .14 & .02 \\
& Dif $120-210$ & $.60^{* * *}$ & $.74^{* * *}$ \\
& Dif $130-210$ & $.46^{* * *}$ & $.14^{* *}$ \\
\hline Difference between & $\mathrm{G}(120)$ & $.18^{* *}$ & .21 \\
First and Final Part & $\mathrm{G}(130)$ & .09 & -.09 \\
\hline \hline
\end{tabular}

Notes: A matching group observation in a given period is classified as consistent with the separating equilibrium prediction if and only if the acceptance decision was predicted correctly and |equilibrium action - observed action $\mid \leq 10$ for at least 3 of the 5 pairs in the matching group; the middle column displays the fraction of equilibrium observations given that the previous observation was not in equilibrium; the right column displays the fraction of equilibrium observations given that the previous observation was in equilibrium.

Summarizing, the two main features of the dynamics in the data are $(i)$ there is language-inflation and (ii) the separating equilibrium attracts behavior over time in $\mathrm{G}(120)$ and to a slightly lesser extent in $\mathrm{G}(130)$, but not at all in $\mathrm{G}(210)$.

\subsection{Best Response Dynamic}

A first avenue to look to explain the data is a simple best response model. This, however, cannot distinguish between the two equilibria or the effects of $B$ using natural initial conditions. (We get equivalent results for a level- $k$ analysis.) To see why, consider the simplest best response dynamic, in which Sender and Receiver best respond to the other's strategy in the previous period. We again assume that Senders induce the action they prefer most. If Senders are indiffer- 
ent, they randomize between their optimal actions. The outcome of the best response model depends very much on the initial conditions and we will look at the two natural starting points: a babbling strategy and a naive strategy. In the babbling strategy no information is transmitted: Senders randomize in the interval $[0, B]$ and Receivers take the optimal prior action 45 regardless of the message (this corresponds to a random level-0 in a level- $k$ analysis). In the naive strategy all information is transmitted: Senders report their type and this is believed by Receivers (this corresponds to a truthful level-0 in a level- $k$ analysis). It is readily verified that if players babble in the first period, then the dynamic forever stays in this pooling equilibrium, regardless of the boundary. Similarly, if players use a naive strategy in the first periods, it can be shown that the dynamic converges to the separating equilibrium, regardless of the boundary.

\subsection{Neologism Dynamic}

We introduce a small twist to create a 'neologism dynamic': Sender types who can send a credible neologism with respect to the Receiver's strategy in the previous round will do so and such a credible neologism will be believed. In all other respects, the dynamic is the same as above. If we analyze this dynamic for our experimental treatments $(\mathrm{G}(120), \mathrm{G}(130), \mathrm{G}(210))$, we get entirely different results. First, the outcome becomes less dependent on the initial conditions. Second, the dynamic converges to behavior that resembles the separating equilibrium when the ACD is small and only when it is small. ${ }^{23}$ Finally, in the attractor of $\mathrm{G}(130)$ and $\mathrm{G}(210)$, types close 30 do not separate neatly as they would in the separating equilibrium. (A level- $k$ with neologisms analysis yields qualitatively the same result as the best response dynamic.)

In $\mathrm{G}(120)$, the dynamic converges to a steady state that corresponds to the separating equilibrium for both random and naive first-period strategies. If players have a naive strategy in period 1, then Senders realize in period 2 that

\footnotetext{
${ }^{23}$ Although (in line with ACDC) the dynamic converges in all cases to behavior that is closer to the separating than to the pooling equilibrium.
} 
they should send their type plus 60, leading to an inflation of language. Types higher than 60 pool at the highest message of 120 . In period 3, Receivers recognize the language inflation and propose 0 to any message smaller than 120 . In addition, they propose 60 if they receive 120 . In period 4, the players are already in the separating equilibrium. Note that as long as the Receiver proposes 0 and an action higher or equal to 60, no neologism can be played. Suppose players start with a babbling strategy. Then in period 2, Senders in $[0,22.5)$ send a low neologism of 0 and Senders in $(57.5,120]$ send a high neologism of 60. In period 3, the Receiver realizes that types who do not send a neologism accept 0 , and propose 0 to them and 60 to others. As a result, in period 4 equilibrium is reached.

In $\mathrm{G}(130)$, the dynamic starts out (for both initial conditions) similar to G(120) but does not converge to the separating equilibrium. Instead, the dynamic converges to a four-cycle that, nonetheless, stays pretty close to the separating equilibrium. In $\mathrm{G}(210)$, the dynamic converges to a (non-steady) state, where the Receiver proposes actions 0, 30 and 90, and the Senders in $(90,210]$ send a neologism of 90 . Hence, the dynamic does not come close to the separating equilibrium. (Although it comes closer to the separating than to the pooling equilibrium.) We summarize the findings in Figure 5. We have held the discussion of the neologism dynamic informal here. For details and proofs, we refer to Appendix B. The calculations are straightforward, but tedious.

Observe that the results of the neologism dynamic are in line with the two main dynamic features of the experiment: $(i)$ there is language-inflation and $(i i)$ the separating equilibrium attracts behavior in $\mathrm{G}(120)$ and to a slightly lesser extent in G(130), but not at all in G(210). (See Figure 4.) Furthermore, the observation that the prediction error of the separating equilibrium decreases over time in $\mathrm{G}(120)$ and $\mathrm{G}(130)$, but not in $\mathrm{G}(210)$ is in line with the neologism dynamic. Finally, note that in G(130) and G(210) types close to 30 (the indifference type in the separating equilibrium) do not separate neatly into low and high types. Hence, the dynamic predicts a messy separation close to the equilibrium indifference type for these treatments, which we observe in the data. In 
addition, it supports the assumption of ACDC that in an equilibrium with a small ACD, behavior will not be entirely in equilibrium but can be close to it (and that it can deviate substantially from equilibrium if the ACD is large).

Our neologism dynamic is a parsimonious behavioral model that organizes the main patterns in the data even though it ignores some features that may also play a role. Firstly, it abstracts away from noise, which is present in the data (as is the case in most experiments). Hence, adding noise to our analysis, in the spirit of Quantal Response (McKelvey \& Palfrey, 1995; 1998) would certainly make it more realistic. Nonetheless, in this case formalizing noise would not teach us much more about the data except that agents best respond in a noisy manner. In particular, an Agent Quantal Response (A-QRE) analysis per se cannot (meaningfully) select equilibria in cheap talk games: The pooling equilibrium is always a limiting principal branch A-QRE, while it is often implausible. ${ }^{24}$ Secondly, there is somewhat more separation close to the indifference type than predicted by the separating equilibrium. Although this is predicted by the neologism dynamic predicts when that equilibrium is not neologism proof, other forces may also be in play here. In particular, one could think in the direction of lying aversion or some naivety on the part of the Receivers, such as in the model of Kartik, Ottaviani \& Squintani (2007). ${ }^{25}$

\footnotetext{
${ }^{24}$ The A-QRE is the extensive form game variant of the QRE. The pooling equilibrium of a cheap talk game is always a limiting, principal branch A-QRE: for any rationality parameter $\lambda$, there is an A-QRE where all Senders mix uniformly over the message space and the Receiver ignores all messages. As $\lambda$ increases, the Senders strategy remains unchanged, and the Receiver's best response smoothly approaches its actual best response to her prior.

${ }^{25}$ In De Groot Ruiz, Offerman and Onderstal (2011), we also studies the degree of information transmission in treatments G(120), T4 and T5. The information contained in messages is very close to that predicted by equilibrium, although actual information transmission is a bit lower, since Receivers do not manage to decode all information. Our results are thus closer to those of Agranov \& Schotter (2012). who observe the 'right' amount of information transmission in a coordination game, than to those of Cai \& Wang (2006) who observe overcommunication in a Crawford-Sobel setting.
} 

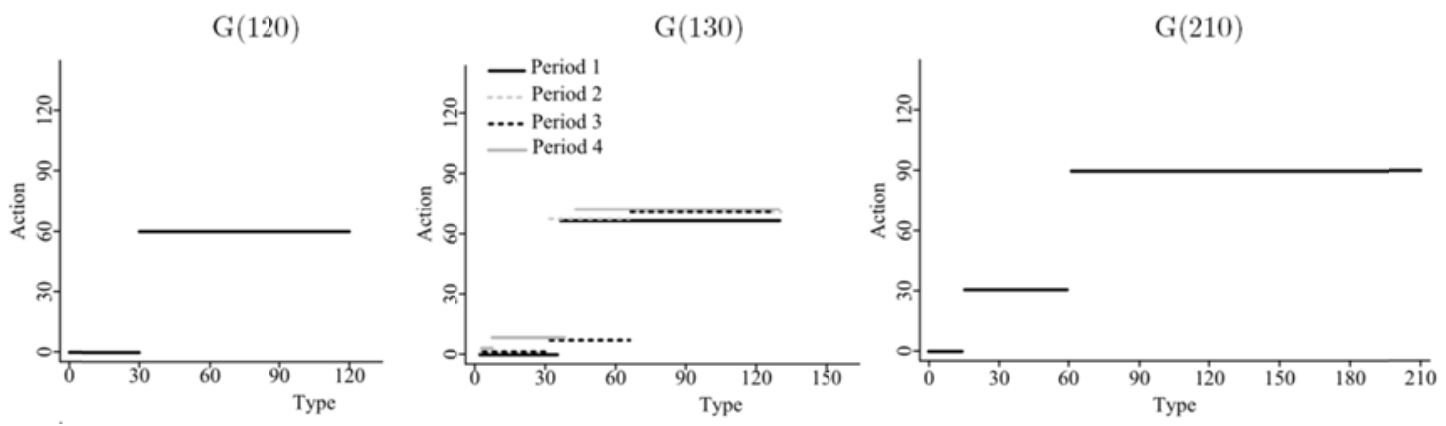

FIGURE 5

The figure shows the type-action plots the attractors of the neologism dynamics for boundaries $G(120), G(130)$ and $G(210)$. In $G(120)$, the attractor is a steady state profile equal to the separating equilibrium. In $\mathrm{G}(130)$, the attractor is a four-cycle characterized by Receiver actions $\left\{0,64 \frac{2}{7}\right\} ;\left\{0,64 \frac{2}{7}, 70\right\} ;\left\{0,7 \frac{1}{7}, 70\right\}$ and $\left\{0,7 \frac{1}{7}, 64 \frac{2}{7}\right\}$. In $\mathrm{G}(210)$ the attractor is the strategy profile $\{0,30,90\}$ (which is attracting but not a steady state).

\section{Robustness}

We ran two additional treatments, $\mathrm{T} 4$ and $\mathrm{T} 5$, to perform two types of robustness checks. In T4 (and T5), we check that the treatment effects are not due to the increase in the action and type space. In T5, we test ACDC in a more complex setting and compare it with NITS and influentiality. As in section 4, we focus on the last 15 periods and we also use the same statistical methods and notation as in that section.

\section{1. $\mathrm{T} 4$}

One possible experimental risk of manipulating $B$ in treatments $\mathrm{G}(120)$, $\mathrm{G}(130)$ and $\mathrm{G}(210)$ is that our results could be influenced (merely) by increasing the action and type space. In particular, one may be worried that confused subjects simply choose something close to the midpoint of the message or action space. Therefore, we ran T4, which is strategically equivalent to the game $\mathrm{G}(240)$, but where we do not shift the boundary (we keep it at 120), but change the disagreement point payoff instead. T4 is identical to $G(120)$, except that disagreement payoff for both players is 30 : $U^{R}(\delta)=U^{S}(\delta)=30$. We have: 
Proposition 4 T4 has a size-1 equilibrium $\left\{a_{1}=\frac{45}{2}\right\}$ and a size-2 equilibrium $\left\{a_{1}=0, a_{2}=30\right\}$. In addition, $\mathrm{T} 4$ is strategically equivalent to $\mathrm{G}(240)$.

Figure 6 shows a type-action bubble plot of T4 (left panel) and Table 5 shows the ACDC properties of T4 together with those of $\mathrm{G}(120), \mathrm{G}(130)$ and $\mathrm{G}(210)$. The results of $\mathrm{T} 4$ are in line with those of the three $\mathrm{G}(B)$ treatments. In particular, the data of $\mathrm{T} 4$ are close to $\mathrm{G}(210)$, except that outcomes in $\mathrm{T} 4$ are even more dispersed, which is in line with the higher ACD of the separating equilibrium.
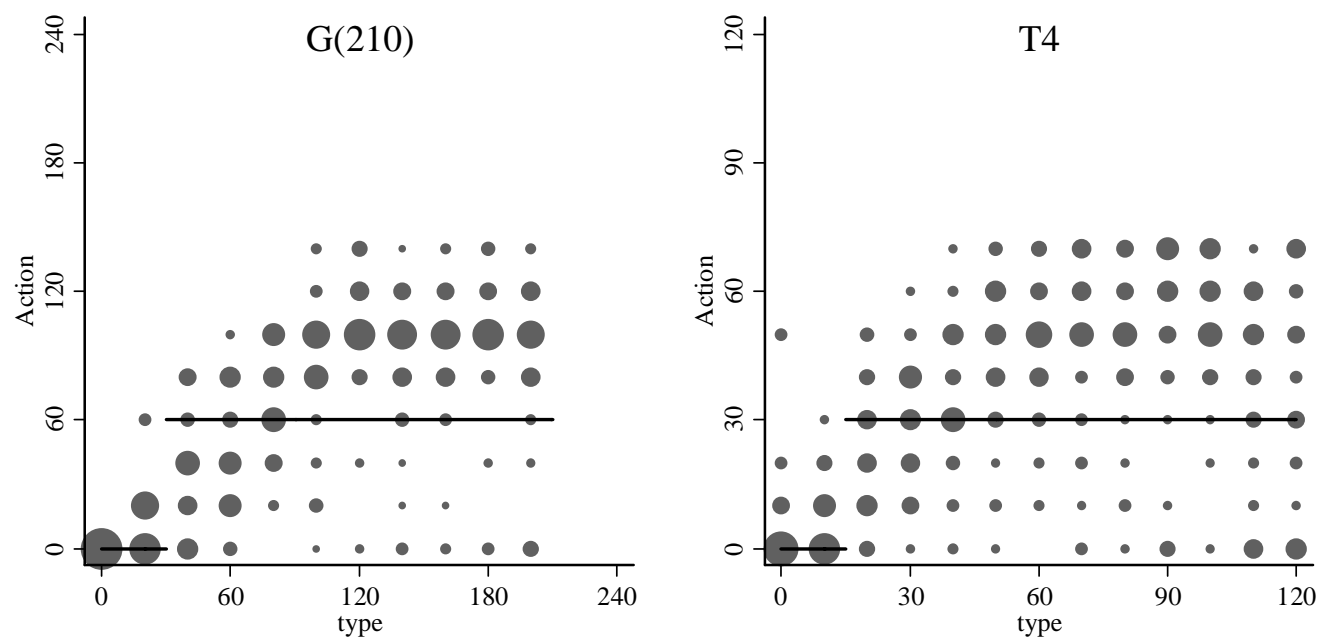

FiguRE 6

This figure shows the type action bubble plot for $\mathrm{G}(210)$ and T4. The bubble plots are clustered on a grid of 10 . The solid line represents the ACDC equilibrium outcome.

Furthermore, note that the average high action is close to the middle of the action space in $\mathrm{G}(120)$ and $\mathrm{G}(130)$. Low types do not induce actions close the midpoint in any treatment, so the midpoint does not drive results. Still, it could be that the midpoint is in some way focal for high actions. T4 and T5 (which we discuss in the subsequent subsection) show that this is not the case. In both $\mathrm{T} 4$ and $\mathrm{T} 5$, the midpoint is 60 as in $\mathrm{G}(120)$, but in $\mathrm{T} 4$ the average high action is 49 and in T5 the average high action is 79. (See Figure 7 for a plot of T5.) 
TABLE 5

Performance Pooling Versus (ACDC) Separating Equilibrium

\begin{tabular}{lccccc}
\hline \hline & \multicolumn{2}{c}{ ACD } & \multicolumn{3}{c}{ Average Prediction Error } \\
\hline & Pooling & Separating & Pooling & Separating & Difference \\
\hline $\mathrm{G}(120)$ & 0.39 & 0 & 18.3 & 10.7 & $7.5^{* *}$ \\
$\mathrm{G}(130)$ & 0.45 & 0.22 & 22.7 & 12.5 & $10.2^{* *}$ \\
$\mathrm{G}(210)$ & 0.63 & 0.50 & 42.3 & 33.3 & $9.0^{* *}$ \\
$\mathrm{~T} 4 \sim \mathrm{G}(240)$ & 0.65 & 0.56 & 46.6 & 37.6 & $4.5^{* *}$ \\
\hline \hline
\end{tabular}

Notes: This table shows the theoretical ACD and the observed prediction error of each equilibrium. The prediction error of $\mathrm{T} 4$ is scaled (doubled) to make it comparable with the other treatments.

\subsection{T5}

There are a few issues we cannot test in $\mathrm{G}(B)$. First, we cannot test whether ACD also organizes data in a more complicated setting, where the equilibrium set is large. Secondly, our conjecture that credible deviations matter gradually implies that, even if a neologism proof equilibrium exists, other equilibria admitting few credible deviations may not perform that much worse. Finally, in $\mathrm{G}(B)$ we cannot discriminate between ACDC on the one hand and NITS and influentiality on the other in terms of selection properties. ${ }^{26}$ For these two reasons, we study T5.

T5 is the same as G(120), except that the Sender's disagreement point payoff is raised from 0 to 30 : $U^{R}(\delta)=0, U^{S}(\delta, t)=30$. In T5, the maximum equilibrium-size is 3 and continua of equilibria exist:

Proposition 5 T5 has a size-1 equilibrium $\left\{a_{1}=30\right\}$. In addition, it has a set of size-2 respectively size-3 equilibria characterized by $\left\{a_{1}, a_{2}=a_{1}+60\right\}$, with $a_{1} \in[0,30]$ and $\left\{a_{1}=0, a_{2}, a_{3}=a_{2}+60\right\}$ with $a_{2} \in(0,30]$. The ACDC equilibrium, which is also neologism proof, is $\{0,30,90\}$.

Observe that all size-3 equilibria survive the influentiality criterion. The size-2 equilibrium $\{0,60\}$ and all size-3 equilibria survive NITS (relative to lowest type

\footnotetext{
${ }^{26}$ The influentiality criterion selects those equilibria with the highest number of actions.
} 
$t=0)$ : the lowest type has no incentive to separate, because it obtains its highest possible utility in equilibrium.

As Figure 7 shows, in T5, behavior roughly follows the predictions of the ACDC equilibrium, although there is excess separation. Primarily, types close to the boundaries between the intervals of the ACDC equilibrium tend to elicit different actions than in equilibrium.

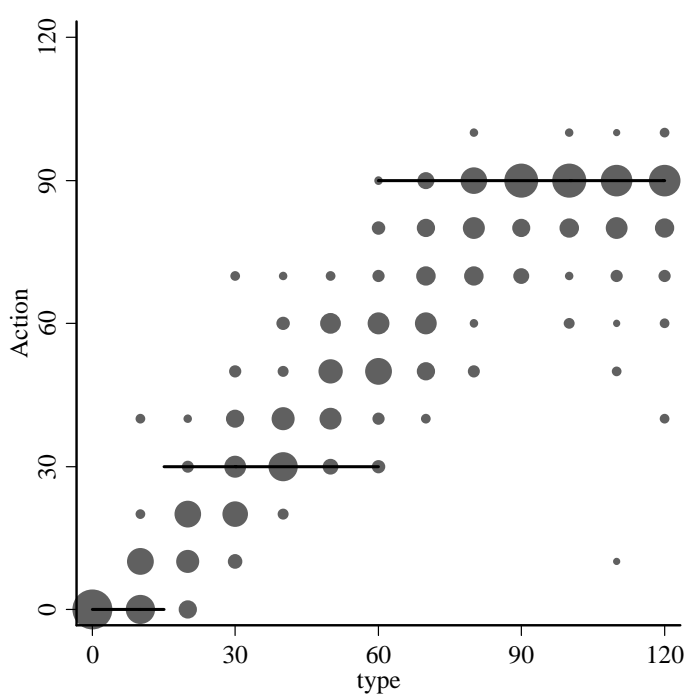

FiguRE 7

This figure shows the type action bubble plot for T5. The bubble plots are clustered on a grid of 10 . The solid line represents the ACDC equilibrium outcome.

The ACDC equilibrium predicts significantly better than the pooling equilibrium. (The prediction error of the separating equilibrium (11.4) is significantly smaller at the $5 \%$ level than that of the pooling equilibrium (34.3).) Furthermore, Figure 8 shows that in T5 the ACDC equilibrium outperforms the other equilibria as well. The left hand side displays the theoretical ACD for the size-2 and size-3 equilibria. The equilibrium that is characterized by the actions $(0,30,90)$ minimizes the ACD and is thus the ACDC equilibrium. The right hand side of the figure shows that for this equilibrium the average prediction error of the action is minimized. In addition, the rank of an equilibrium's prediction error (right panel in Figure 8) roughly follows the rank an equilibrium's ACD (left panel in Figure 8). (The two plots have a different curvature though.) This 
is also interesting since the ACDC equilibrium is neologism proof. Equilibria that are not neologism proof, but have a small ACD perform quantitatively but not qualitatively worse. In sum, ACDC organizes the data quite well.

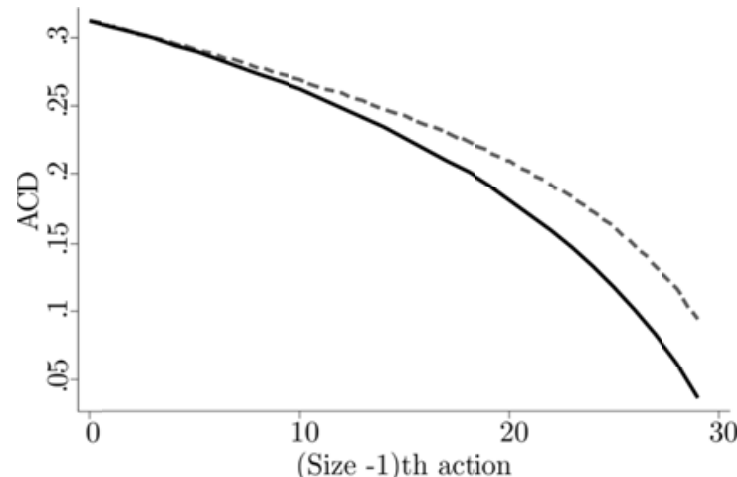

----- Size 2 Equilibria $\longrightarrow$ Size 3 equilibria

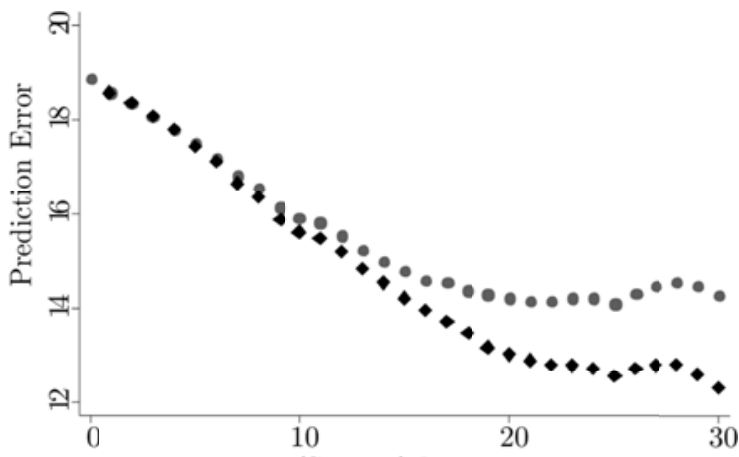

(Size - 1)th action

FIGURE 8

ACD and performance of size- 2 and size- 3 equilibria in T5. The left panel plots the theoretical ACD of the size- 2 and sieze-3 equilibria, whereas the right panel plots the average prediction error of the equilibria. These equilibria can be characterized by $a_{\text {size- } 1}$. There are 31 size-2 equilibria of the form $\left(a_{1}, a_{1}+60\right)$ with $0 \leq a_{1} \leq 30$ and 30 size- 3 equilibria of the form $\left(0, a_{2}\right.$, $\left.a_{2}+60\right)$ with $0<a_{1} \leq 30$.

\section{Conclusion}

In this paper we presented an experimental test of ACDC. Our main conclusion is that the results provide systematic support for ACDC: credible deviations matter and they matter gradually. In addition, we find that a neologism dynamic can organize the main dynamic characteristics of the data. In De Groot Ruiz, Offerman \& Onderstal (2012a), we show that ACDC performs at least as well as existing criteria in previously conducted experiments. The current study supports the predictions of ACDC in a new setting where existing concepts are silent. 


\section{Appendix A: Proofs of Propositions}

Proofs of Propositions 1, 4 and 5

$\mathrm{G}(B)$, T4 and T5 belong to the following game $\Gamma\left(d^{R}, d^{S}\right)$, which is the uniform linear case of the veto threats model we study in De Groot Ruiz, Offerman \& Onderstal (2012b). $\Gamma\left(d^{R}, d^{S}\right)$ proceeds as game $\mathrm{G}(B)$. However, the Sender's type $t$ is drawn from the interval $[0,1]$. The Receiver's and Sender's payoff on the real line are given by $U^{R}(x)=-x$ and $U^{S}(x, t)=-|x-t|$. The disagreement point payoff is $U^{R}(\delta)=-d^{R}$ and $U^{S}(\delta)=-d^{S}$ with $d^{R}, d^{S}>0$. In De Groot Ruiz, Offerman \& Onderstal (2012b), we show that the equilibria of $\Gamma\left(d^{R}, d^{S}\right)$ can be characterized as follows:

Lemma 1 Let $\bar{x}=\max \left\{0, \min \left\{d^{R}-2 d^{S}, 1-d^{S}\right\}\right\}$. Any equilibrium of the game is a partition equilibrium that can be described by a natural number $n \in\{1, \ldots, \bar{n}\}$ and a set of equilibrium actions $\left\{a_{1}, \ldots, a_{n}\right\}$, such that

(i) $\quad a_{1}=\max \left\{0, \min \left\{1-d^{S}, d^{S}, \frac{1}{2}\left(d^{R}-d^{S}\right)\right\}\right\}$ if $n=1$

(ii) $\quad a_{1}=\min \left\{d^{S}, \max \left\{0, a_{2}-2 d^{S}\right\}\right\}$ if $n \geq 2$

(iii) $\quad a_{2}=\min \left\{\frac{2}{3}\left(d^{R}-d^{S}\right), 2 d^{S}, 1-d^{S}\right\}$ if $n=2$ and $a_{1}=0$

(iv) $a_{k}=a_{k-1}+2 d^{S}$ if $a_{k-1}$ exists and $a_{k-1}>0$

(v) $\quad a_{n} \leq \bar{x}$ if $d^{R} \geq 4 d^{S}$

The maximum size $\bar{n}$ is equal to 1 if $d^{S} \geq 1$. If $d^{S}<1, \bar{n}=\max \left\{2,\left[\frac{d^{R}}{2 d^{S}}\right]\right\}$ if $d^{R} \leq d^{S}+1$ and $\bar{n}=\max \left\{2,\left[\frac{3}{2}+\frac{1}{2 d^{S}}\right]\right\}$ otherwise, where $\lceil$.$\rceil is the ceiling$ function. 
From Proposition 5 in (the Online Appendix of) De Groot Ruiz, Offerman \& Onderstal (2012a), it follows that

Lemma 2 The unique $A C D C$ equilibrium of $\Gamma\left(d^{R}, d^{S}\right)$ with respect to credible neologisms is the maximum size equilibrium with the highest equilibrium action.

$\mathrm{G}(B)$ corresponds to $\Gamma\left(d^{R}, d^{S}\right)$ with $d^{R}=\frac{5}{4} \frac{120}{B}$ and $d^{S}=\frac{1}{2} \frac{120}{B}$. T4 corresponds to $\Gamma\left(\frac{5}{8}, \frac{1}{4}\right)$ and T5 to $\Gamma\left(\frac{5}{4}, \frac{1}{4}\right)$. Propositions 1,4 and 5 are direct corollaries of Lemmas 1 and 2 .

Proofs of Propositions 2 and 3

Proof of Proposition 2 Let $a(t)$ characterize the equilibrium outcome. In our game, $\langle\tilde{a},[\underline{\tau}, \bar{\tau}]\rangle$ is a credible neologism iff $U^{S}(\tilde{a}, t)<U^{S}(a(t), t) \forall t \notin[\underline{\tau}, \bar{\tau}]$, $U^{S}(\tilde{a}, t)>U^{S}(a(t), t) \forall t \in(\underline{\tau}, \bar{\tau})$ and $\tilde{a}=a^{*}[\underline{\tau}, \bar{\tau}]$. Hence $\tilde{a}<a_{1}$ implies $\underline{\tau}=0$ and $\tilde{a}>a_{n}$ implies $\underline{\tau}=B$.

First, let us look at pooling equilibrium $\sigma^{P}$. Consider a low credible neologism $\tilde{a}^{L}<a=45$. Now, $\underline{\tau}^{L}=0$. Furthermore, $\bar{\tau}^{L}=\frac{1}{2}\left(\bar{a}^{L}+45\right)<60$. Hence, $\tilde{a}^{L}=a^{*}[0, \bar{\tau}]=0$ and $\bar{\tau}^{L}$ must be 22.5. Next, consider a high credible neologism $\tilde{a}^{H}>45, \bar{\tau}^{H}=B$ and $\underline{\tau}^{H}=\frac{1}{2}\left(\bar{a}^{H}+45\right)$. Solving $a^{*}\left[\frac{1}{2}\left(\bar{a}^{H}+45\right), B\right]=\tilde{a}^{H}$ yields $\tilde{a}^{H}=\min \{B-60,75\}>45$. Consequently, $\underline{\tau}^{H}=\min \left\{\frac{B-15}{2}, 60\right\}$.

Second, let us look at the separating equilibrium $\sigma^{S}$. There can be no credible equilibrium $\tilde{a}<a_{1}$ as $a_{1}=0$. Consider a credible neologism $\tilde{a}>60$. Now, $\bar{\tau}=\quad B \quad$ and $\quad \underline{\tau}=\frac{1}{2}(d+60) . \quad$ Solving $\quad a^{*}\left[\frac{1}{2}(d+60), B\right]=\tilde{a} \quad$ yields $\quad \tilde{a}=$ $\min \{B-60,80\}$. Hence, $\underline{\tau}=\min \left\{\frac{B}{2}, 70\right\}$. If $B=120$, then $\tilde{a}=60$ and it is no neologism. If $B>120$, it is a neologism. Finally, consider some neologism with 
$a_{1}=0<\tilde{a}<a_{2}=60$. Since $\tilde{a}<60$, it must be that $\bar{\tau}<60$. However, if $\bar{\tau}<60$, then $a^{*}[\underline{\tau}, \bar{\tau}]=0$. Hence $\tilde{a}$ cannot be a neologism. Q.E.D.

Proof of Proposition 3 First, we show that $A C D\left(\sigma^{P}\right)>A C D\left(\sigma^{S}\right)$. Let $\underline{\tau}^{H}$ be the lowest deviating type of the high neologism in the pooling equilibrium $\sigma^{P}$ and $\underline{\tau}$ the lowest deviating type of the neologism in the separating equilibrium $\sigma^{S}$. Due to the low credible neologism, $C D\left(t, \sigma^{P}\right)>C D\left(t, \sigma^{S}\right)=0$ for $t \in\left[0, \frac{45}{2}\right)$. For $t \in\left[\frac{45}{2}, \underline{\tau}^{H}\right), \quad C D\left(t, \sigma^{P}\right)=C D\left(t, \sigma^{S}\right)=0$. Since the distance between the neologism action and the equilibrium action is larger in the pooling equilibrium than in the separating equilibrium and $\underline{\tau}^{H}<\underline{\tau}$, it must hold that $C D\left(t, \sigma^{P}\right)>C D\left(t, \sigma^{S}\right)$ for $t \in\left(\underline{\tau}^{H}, 120\right]$. Furthermore, $C D\left(t, \sigma^{P}\right)=C D\left(t, \sigma^{S}\right)=1$ for $t \in[120, B]$. Hence, $E_{t}\left[C D\left(t, \sigma^{P}\right)\right]>E_{t}\left[C D\left(t, \sigma^{S}\right)\right]$.

For the second result, observe that the set of rationalizable actions for the Receiver is $[0, B-60]$ and that the Sender can always guarantee herself a payoff of 0 by rejecting the proposed action. This means that $\bar{U}^{S}(t)=U^{S}(\min \{t, B-60\}, t) \quad$ and $\quad \underline{U}^{S}(t)=\max \left\{0, \min \left\{U^{S}(0, t), \quad U^{S}(B-60, t)\right\}\right\}$. Using Proposition 1 and Proposition 2, we get for the ACD of the separating equilibrium

$$
A C D\left(\sigma^{S}\right)=\frac{1}{B} \int_{\min \{70, B / 2\}}^{120} \frac{\{(60-\mid t-(\min \{80, B-60\} \mid)-(60-|t-60|)\}}{\bar{U}^{S}(t)-\underline{U}^{S}(t)} d t
$$

$+\frac{B-120}{B}$. It is readily verified that this function is zero for $B=120$ and strictly increasing in $\mathrm{B}$ for $B \geq 120$. Q.E.D. 


\section{References}

Agranov, M., \& Schotter, A. (2011). Language and Leadership: an Experimental Study of Ambiguity and Vagueness in the Announcement Game. Working paper, New York University.

Agranov, M., \& Schotter, A. (2012). Ignorance is Bliss: an Experimental Study of the Use of Ambiguity and Vagueness in the Coordination Games with Asymmetric Payoffs. AEJ: Microeconomics, 4, 77-103.

Blume, A., \& Sobel, J. (1995). Communication-Proof Equilibria in Cheap-Talk games. Journal of Economic Theory, 65, 359-382.

Blume, A., DeJong, D. V., Kim, Y.-G., \& Sprinkle, G. B. (1998). Experimental Evidence on the Evolution of Meaning of Messages in Sender-Receiver Games. American Economic Review, 88, 1323-1340.

Blume, A., DeJong, D. V., Kim, Y.-G., \& Sprinkle, G. B. (2001). Evolution of Communication with Partial Common Interest. Games and Economic Behavior, 37, 79-120.

Blume, A., Kim, Y.-G., \& Sobel, J. (1993). Evolutionary Stability in Games of Communication. Games and Economic Behavior, 5, 547-575.

Cai, H., \& Wang, J. T. (2006). Overcommunication in Strategic Information Transmission Games. Games and Economic Behavior, 56, 7-36.

Cason, T. N. (1995). Cheap talk Price Signaling in Laboratory markets. Information Economics and Policy, 7, 183-204.

Charness, G. (2000). Self-Serving Cheap Talk: A Test Of Aumann's Conjecture. Games and Economic Behavior, 33, 177-194.

Charness, G., \& Dufwenberg, M. (2006). Promises and Partnership. Econometrica, 74, 1579-1601.

Chen, Y., Kartik, N., \& Sobel, J. (2008). Selecting Cheap Talk Equilibria. Econometrica, 76, 117-136.

Coles, P., Kushnir, A., \& Niederle, M. (Forthcoming). Preference Signaling in Matching Markets. AEJ: Microeconomics.

Crawford, V. (1998). A Survey of Experiments on Communication via Cheap Talk. Journal of Economic Theory, 78, 286-298. 
Crawford, V., \& Sobel, J. (1982). Strategic Information Transmission. Econometrica, 50, 1431-1451.

Croson, R., Boles, T., \& Murnighan, J. K. (2003). Cheap Talk in Bargaining Experiments: Lying and Threats in Ultimatum Games. Journal of Economic Behavior \& Organization, 51, 141-159.

Daniel, T. E., Seale, D. A., \& Rapoport, A. (1998). Strategic Play and Adaptive Learning in the Sealed-Bid Bargaining Mechanism. Journal of Mathematical Psychology, 42, 133-166.

Davis, D. D., \& Holt, C. A. (1988). Conspiracies and Secret Discounts in Laboratory Markets. Economic Journal, 108, 736-756.

De Groot Ruiz, A. W., Offerman, T., \& Onderstal, S. (2011). An Experimental Study of Credible Deviations and ACDC: Extended Version. Working paper, University of Amsterdam.

De Groot Ruiz, A. W., Offerman, T., \& Onderstal, S. (2012a). Equilibrium Selection in Cheap Talk Games: ACDC Rocks When Other Criteria Remain Silent. Working paper, University of Amsterdam.

De Groot Ruiz, A. W., Offerman, T., \& Onderstal, S. (2012b). Power and the Privilege of Clarity: An Analysis of Bargaining Power and Information Transmission. Working paper, University of Amsterdam.

DellaVigna, S., \& Gentzkow, M. (2010). Persuasion: Empirical Evidence. Annual Review of Economics, 2, 643-669.

Dickhaut, J. W., McCabe, K. A., \& Mukherji, A. (1995). An Experimental Study of Strategic Information Transmission. Economic Theory, 6, 389403.

Ellingsen, T., \& Johannesson, M. (2004). Promises, Threats and Fairness. The Economic Journal, 114, 397-420.

Farrell, J. (1993). Meaning and Credibility in Cheap-Talk Games. Games and Economic Behavior, 5, 514-531.

Farrell, J., \& Gibbons, R. (1989). Cheap Talk can Matter in Bargaining. Journal of Economic Theory, 48, 221-237.

Fischbacher. (2007). z-Tree: Zurich Toolbox for Ready-made Economic Experiments. Experimental Economics, 10, 171-178. 
Forsythe, R., Kennan, J., \& Sopher, B. (1991). An Experimental Analysis of Strikes in Bargaining Games with One-Sided Private Information. American Economic Review, 81, 253-278.

Gilligan, T. W., \& Krehbiel, K. (1990). Organization of Informative Committees by a Rational Legislature. American Journal of Political Science, 34, 531564.

Gneezy, U. (2005). Deception: The Role of Consequences. American Economic Review, 95, 384-394.

Kartik, N., Ottaviani, M., \& Squintani, F. (2007). Credulity, Lies and Costly Talk. Journal of Economic Theory, 134, 93-116.

Kohlberg, E., \& Mertens, J.-F. (1986). On the Strategic Stability of Equilibria. Econometrica, 54, 1003-1037.

Lundquist, T., Ellingsen, T., Gribbe, E., \& Johannesson, M. (2009). The Aversion to Lying. Journal of Economic Behavior \& Organization, 70, 8192.

Matthews, S. A. (1989). Veto Threats: Rhetoric in a Bargaining Game. Quarterly Journal of Economics, 104, 347-369.

Matthews, S. A., \& Postlewaite, A. (1989). Pre-Play Communication in TwoPerson Sealed-Bid Double Auctions. Journal of Economic Theory, 48, 238-263.

Matthews, S. A., Okuno-Fujiwara, M., \& Postlewaite, A. (1991). Refining Cheap-Talk Equilibria. Journal of Economic Theory, 55, 247-273.

McKelvey, R. D., \& Palfrey, T. R. (1995). Quantal Response Equilibrium for Normal Form Games. Games and Economic Behavior, 10, 6-38.

McKelvey, R. D., \& Palfrey, T. R. (1998). Quantal Response Equilibrium for Extensive Form Games. Experimental Economics, 1, 9-41.

Miller, R. M., \& Plott, C. A. (1985). Product Quality Signaling in Experimental Markets. Econometrica, 53, 837-872.

Morgan, J., \& Stocken, P. C. (2003). An Analysis of Stock Recommendations. The RAND Journal of Economics, 34, 183-203.

Morris, S. (2001). Political Correctness. Journal of Political Economy, 109, 231265.

Myerson, R. B. (1989). Credible Negotiation Statements and Coherent Plans. Journal of Economic Theory, 48, 264-303. 
Oosterbeek, H., Sloof, R., \& Van der Kuilen, G. (2004). Cultural Differences in Ultimatum Game Experiments: Evidence from a Meta-Analysis. Experimental Economics, 7, 171-188.

Rabin, M. (1990). Communication between Rational Agents. Journal of Economic Theory, 51, 144-170.

Rabin, M., \& Sobel, J. (1996). Deviations, Dynamics and Equilibrium Refinements. Journal of Economic Theory, 68, 1-25.

Radner, R., \& Schotter, A. (1989). The Sealed Bid Mechanism: An Experimental Study. Journal of Economic Theory, 48, 179-220.

Rapoport, A., Erev, I., \& Zwick, R. (1995). An Experimental Study of BuyerSeller Negotiation with One-Sided Incomplete Information and Time Discounting. Management Science, 41, 377-394.

Schotter, A., Snyder, B., \& Zheng, W. (2000). Bargaining through Agents: An Experimental Study. Games and Economic Behavior, 30, 248-292.

Selten, R. (1975). Reexamination of the Perfectness Concept for Equilibrium Points in Extensive Games. International Journal of Game Theory, 4, 2555.

Serra-Garcia, M., Van Damme, E., \& Potters, J. (2011). Lying About What You Know or About What You Do? TILEC Discussion paper, University of Tilburg.

Valley, K. L., Moag, J., \& Bazerman, M. H. (1998). A Matter of Trust: Effects of Communication on the Efficiency and Distribution of Outcomes. Journal of Economic Behavior \& Organization, 34, 211-238.

Wang, J. T., Spezio, M., \& Camerer, C. F. (2010). Pinocchio's Pupil: Using Eyetracking and Pupil Dilation to Understand Truth Telling and Deception in Sender-Receiver Games. American Economic Review, 100, 984-1007. 


\section{Appendix B (Online): Neologism Dynamic}

We first describe the standard, simple, best response dynamic. In each period all Sender types and the Receiver choose a strategy. We assume that the Sender in the acceptance stage accepts all actions that yield her nonnegative payoff: $\nu(a, t)=1$ if $U^{S}(a, t) \geq 0$ and $\nu(a, t)=0$ otherwise. The strategy of the Sender in period $r$ is then given by $\mu_{r}: T \rightarrow \Delta M$ and that of the Receiver by $\alpha_{r}: M \rightarrow A$. Let $m_{r}(t)$ denote the message Sender type $t$ sends (which may be a random variable) and $a_{r}(m)$ denote the Receiver's action after receiving message $m$. Both players best respond to the strategy of the other player in the previous round. First, the support of $\mu_{r}(t)$ is equal to $\arg \max _{m \in M} U^{S}\left(a_{r-1}(m), t\right)$.

In particular, we assume the Sender randomizes uniformly over the set of best responses. Second, $a_{r}(m)=\arg \max _{a \in A} U^{R}(a, t) E_{t}\left[\nu(a, t) \mid \beta^{r}(m)\right]$, where $\beta^{r}(m)$ is derived from $\mu_{r-1}$ by Bayes rule whenever possible. ${ }^{27}$ If $\beta^{r}$ cannot be derived from $\mu_{r-1}$, then $\beta^{r}=\beta^{r}(m)$ for some randomly chosen $m \in \cup_{t \in T} \operatorname{supp} \mu_{r-1}(t)$.

The neologism dynamic differs from the best response dynamic on one crucial aspect: Senders can send credible neologisms, which will be believed. We define $\langle\tilde{a}, N\rangle$ as a credible neologism with respect to Receiver strategy $\alpha_{r}$ if (i) $U^{S}(\tilde{a}, t)>\arg \max _{m \in M} U^{S}\left(a_{r}(m), t\right) \cdot \nu\left(a_{r}(m), t\right) \quad$ for $\quad$ all $\quad t \in N$,

$U^{S}(\tilde{a}, t) \leq \arg \max _{m \in M} U^{S}\left(a_{r}(m), t\right) \cdot \nu\left(a_{r}(m), t\right) \quad$ for $\quad$ all $\quad t \notin N \quad$ and

$\tilde{a}=\arg \max _{a \in A} U^{R}(a, t) E_{t}[\nu(a, t) \mid t \in N] .^{28}$

\footnotetext{
${ }^{27}$ We assume (for ease of exposition) that there is one unique best response for the Receiver, which is generically the case in our game. In case there are more optimal actions one could let the Receiver randomize.

${ }^{28}$ We need to point out the following subtlety. If a credible neologism was used in the previous period, it becomes just a message (which may have acquired a new 'meaning'). If the same credible neologism has to be made in the following period, it cannot be the same literal message, as then it would not be a neologism. Hence, the Sender can add for instance Really! or Really, Really! etc. to make it a neologism and distinguish it from the old message.
} 


\section{FOR ONLINE PUBLICATION}

Now, in the neologism dynamic all Senders that can send a credible neologism in round $r$ with respect to $\alpha_{r-1}$, will do so and such credible neologisms will be believed by the Receivers in round $r$. In all other cases, the dynamic is identical to a best response dynamic. We call the neologism dynamic $f\left(\mu_{r}, a_{r}\right)$.

This best response dynamic bears similarities to a level- $k$ analysis. The difference is that in level- $k$, in each iteration just one player (Sender or Receiver) changes her strategy. In the best response dynamic, both players change their strategy each period. Still, the best response dynamic converges in all cases below to very similar outcomes as the outcomes a level- $k$ analysis would converge to.

Before analyzing the dynamic, we characterize the best responses and neologisms. The Sender's best response is simply to induce the action closest to her type. We call the Receiver's best response $a^{*}[\underline{t}, \bar{t}]$ if Sender types are uniformly distributed in the interval $[\underline{t}, \bar{t}] . \quad a^{*}[\underline{t}, \bar{t}]$ is single-valued and equal to $\min \left\{\bar{t}-60,45+\frac{1}{2} \underline{t}\right\}$. Let $\bar{a}=\max _{m \in M}\left\{a_{r}(m)\right\}$ be the highest action of a Receiver's strategy. Then, for $B=120$ and $B=130$, there exists a high credible neologism with respect to $a_{r}$ if and only if $\bar{a}<B-60$. In particular, it is equal to $\quad\left\langle B-60,\left(\frac{B-60+\bar{a}}{2}, 130\right]\right\rangle \quad$ if $\quad 3(B-120) \leq \bar{a}<B-60 \quad$ and $\left\langle 60+\bar{a} / 3,\left(\frac{2}{3}(45+\bar{a}), 130\right]\right\rangle$ if $\bar{a}<3(B-120)$. For $B=210$, there exists a high credible neologism if and only if $\bar{a}<90$, and in this case it is equal to $\left\langle 60+\frac{1}{3} \bar{a},\left(\frac{2}{3}(45+\bar{a}), 130\right]\right\rangle$.

We restrict our analysis to two natural initial strategy profiles: babbling (where no information is transmitted) and naive (where all possible information is transmitted). 


\section{FOR ONLINE PUBLICATION}

For $\mathrm{G}(120), \mathrm{G}(130)$ and $\mathrm{G}(210)$, we (i) give the attractor, ${ }^{29}$ (ii) show that both the babbling and naive initial profiles lie in its basis of attraction and (iii) calculate the average prediction error of the pooling and separating equilibria for the attractor.

$$
G(120)
$$

For $B=120$, it is easy to check that the equilibrium profile is a steady state of the neologism dynamic: $m_{r}(t)=m^{1}$ for $t \in[0,30]$ and $m_{r}(t)=m^{2} \neq m^{1}$ for $t \in[30,120]$, and $a_{r}\left(m^{1}\right)=0$ and $a_{r}\left(m^{2}\right)=60$. It is a steady state of the best response dynamic and no neologism relative to $a_{r}$ exists.

If we start with a babbling profile in period 1, the neologism dynamic proceeds as follows:

\begin{tabular}{|l|l|}
\hline Strategy Sender period 1 (Babbling) & Strategy Receiver period 1 (Babbling) \\
\hline$m_{1}(t) \sim U[0,120]$ if $t \in[0,120]$ & $a_{1}(m)=45$ for all $m \in[0,120]$ \\
\hline
\end{tabular}
where all Senders randomize uniformly over the interval $[1,120]$.

\begin{tabular}{|l|l|}
\hline Strategy Sender period 2 (Babbling) & Strategy Receiver period 2 (Babbling) \\
\hline$m_{2}(t)=n^{1}$ if $t \in[0,45 / 2)$ & $a_{2}(m)=0$ if $m=n^{1}$ \\
\hline$m_{2}(t) \sim U[0,120]$ if $0<t_{6}^{1}=10<15$ & $a_{2}(m)=45$ if $m \in[0,120]$ \\
\hline$m_{2}(t)=n^{2}$ if $t \in(105 / 2,120]$ & $a_{2}(m)=60$ if $m=n^{2}$ \\
\hline
\end{tabular}

where $n^{1}=\langle 0,[0,45 / 2)\rangle$ and $n^{2}=\langle 60,[105 / 2,120)\rangle$

\begin{tabular}{|l|l|}
\hline Strategy Sender period 3 (Babbling) & Strategy Receiver period 3 (Babbling) \\
\hline$m_{3}(t)=n^{1}$ if $t \in[0,45 / 2)$ & $\frac{285}{7} \simeq 40.7$ if $m \in[0,120] \cup\left\{n^{1}\right\}$ \\
\hline$m_{3}(t) \sim U[0,120]$ if $t \in[45 / 2,105 / 2)$ & $a_{3}(m)=60$ if $m=n^{2}$ \\
\hline$m_{3}(t)=n^{2}$ if $t \in[105 / 2,120]$. & \\
\hline
\end{tabular}

\footnotetext{
${ }^{29}$ An attractor is roughly speaking a set in the phase-space the neighborhood of which the dynamic evolves to after sufficient time. This can be, for instance, a steady state or a higher n-
} cycle. 


\begin{tabular}{|l|c|}
\hline Strategy Sender period 4 (Babbling) & Strategy Receiver period 4 (Babbling) \\
\hline$m_{4}(t) \sim U[0,120] \cup\left\{n^{1}\right\}$ if $t \in[0,30)$ & $a_{4}(m)=0$ if $m \in[0,120] \cup\left\{n^{1}\right\}$ \\
\hline$m_{4}(t)=n^{2}$ if $t \in[30,120]$ & $a_{4}(m)=60$ if $m=n^{2}$ \\
\hline
\end{tabular}

Hence, from period 4, the dynamic is and stays in the separating equilibrium.

If we start with a naive profile in period 1, the neologism dynamic proceeds as follows:

\begin{tabular}{|l|l|}
\hline Strategy Sender period 1 (Naive) & Strategy Receiver period 1 (Naive) \\
\hline$m_{1}(t)=t$ if $t \in[0,120]$ & $a_{1}(m)=0$ if $m \in[0,60]$ \\
\hline & $a_{1}(m)=m-60$ if $m \in[60,120]$ \\
\hline
\end{tabular}

where all Senders randomize uniformly over the interval $[1,120]$

\begin{tabular}{|l|l|}
\hline Strategy Sender period 2 (Naive) & Strategy Receiver period 2 (Naive) \\
\hline$m_{2}(t) \sim U[0,60]$ if $t=0$ & $a_{2}(m)=0$ for all $m \in[0,60]$ \\
\hline$m_{2}(t)=t+60$ if $t \in(0,60)$ & $a_{2}(m)=m-60$ for all $m \in[60,120]$ \\
\hline$m_{2}(t)=120$ if $t \in[60,120]$ & \\
\hline
\end{tabular}

\begin{tabular}{|l|l|}
\hline [Strategy Sender period 3 (Naive) & Strategy Receiver period 3 (Naive) \\
\hline$m_{3}(t) \sim U[0,60]$ if $t=0$ & $a_{3}(m)=0$ if $m \in[0,120)$ \\
\hline$m_{3}(t)=t+60$ if $t \in[0,60)$ & $a_{3}(m)=60$ if $m=120$ \\
\hline$m_{3}(t)=120$ if $t \in[60,120]$ & \\
\hline
\end{tabular}

\begin{tabular}{|l|l|}
\hline Strategy Sender period 4 (Naive) & Strategy Receiver period 4 (Naive) \\
\hline$m_{4}(t) \sim U[0,120)$ if $t \in[0,30)$ & $a_{4}(m)=0$ if $m \in[0,120)$ \\
\hline$m_{4}(t)=120$ if $t \in[30,120]$ & $a_{4}(m)=60$ if $m=120$ \\
\hline
\end{tabular}

Hence, from period 4 , the dynamic is and stays in the separating equilibrium.

Now we turn to the prediction error. Let the equilibrium profile be $\sigma^{e}$ and the attracting profile $\sigma^{a}$. Then, the average (or expected) prediction error of an equilibrium for the attracting profile is $E\left[\mid a^{e}\left(m^{e}(t)\right)-a^{a}\left(m^{a}(t)\right) \|\right.$. The average prediction error of the separating equilibrium is obviously 0 . The prediction error of the pooling equilibrium is $\frac{1}{120}\left(\int_{0}^{30}|45-0| d t+\int_{30}^{120}|45-60| d t\right)=45 / 2$. 


\section{FOR ONLINE PUBLICATION}

G(130)

For $B=130$, consider the following state $r^{\prime}$ :

\begin{tabular}{|l|l|}
\hline Strategy Sender period $r^{\prime}$ & Strategy Receiver period $r^{\prime}$ \\
\hline$m_{r^{\prime}}(t)=m^{1}$ if $t \in\left[0, t^{1}\right)$ & $a_{r^{\prime}}(m)=0$ if $m \in\left\{m^{1}, m^{2}\right\}$ \\
\hline$m_{r^{\prime}}(t)=m^{2}$ if $t \in\left[t^{1}, t^{2}\right)$ & $a_{r^{\prime}}(m)=a^{1}$ if $m=m^{3}$ \\
\hline$m_{r^{\prime}}(t)=m^{3}$ if $t \in\left[t^{2}, 130\right]$ & \\
\hline
\end{tabular}

with the restriction that $0 \leq t^{1}<t^{2}<50$ and $50<a 1<70 \cdot m^{1}, m^{2}, m^{3}$ can be any three messages.

Then, by straightforwardly applying the neologism dynamic, we get the following for rounds $r^{\prime}+1, r^{\prime}+2, r^{\prime}+3$ and $r^{\prime}+4$

\begin{tabular}{|l|l|}
\hline Strategy Sender period $r^{\prime}+1$ & Strategy Receiver period $r^{\prime}+1$ \\
\hline$m_{r^{\prime}+1}(t) \sim U\left\{m^{1}, m^{2}\right\}$ if $t \in\left[0, a^{1} / 2\right)$ & $a_{r^{\prime}+1}(m)=0$ if $m \in\left\{m^{1}, m^{2}\right\}$ \\
\hline$m_{r^{\prime}+1}(t)=m^{3}$ if $t \in\left[a^{1} / 2,35+a^{1} / 2\right]$ & $a_{r^{\prime}+1}(m)=45+t^{2} / 4$ if $m=m^{3}$ \\
\hline$m_{r^{\prime}+1}(t)=n^{1}$ if $t \in\left(35+a^{1} / 2,130\right]$ & $a_{r^{\prime}+1}(m)=70$ if $m=n^{1}$ \\
\hline
\end{tabular}

where $n^{1}$ is the credible neologism $\left\langle 70,\left(35+a^{1} / 2,130\right]\right\rangle$. Furthermore, a Sender in $\left[0, a^{1} / 2\right)$, will randomize uniformly over $m^{1}$ and $m^{2}$.

\begin{tabular}{|c|c|}
\hline Strategy Sender period $r+2$ & Strategy Receiver period $r^{\prime}+2$ \\
\hline $\begin{array}{l}m_{r^{\prime}+2}(t) \sim U\left\{m^{1}, m^{2}\right\} \\
t \in\left[0,45 / 2+t^{2} / 4\right)\end{array}$ & $a_{r^{\prime}+1}(m)=0$ if $m \in\left\{m^{1}, m^{2}\right\}$ \\
\hline $\begin{array}{ll}m_{r^{\prime}+2}(t)=m^{3} & \text { if } \\
t \in\left[45 / 2+t^{2} / 4,115 / 2+t^{2} / 4\right) & \end{array}$ & $a_{r^{\prime}+1}(m)=a^{1} / 2-25 \quad$ if $m=m^{3}$ \\
\hline$m_{r^{\prime}+2}(t)=n^{1}$ if $t \in\left[115 / 2+t^{2} / 4,130\right]$ & $a_{r^{\prime}+1}(m)=70$ if $m=n^{1}$ \\
\hline
\end{tabular}

Hence, if player type is in $\left[0, a^{1} / 2\right)$, then she will randomize uniformly over $m^{1}$ and $m^{2}$. 


\begin{tabular}{|c|c|}
\hline Strategy Sender period $r^{\prime}+3$ & Strategy Receiver period $r^{\prime}+3$ \\
\hline $\begin{array}{ll}m_{r^{\prime}+3}(t) \sim U\left\{m^{1}, m^{2}\right\} & \text { if } \\
t \in\left[0, a^{1} / 4-25 / 2\right) & \end{array}$ & $a_{r^{\prime}+3}(m)=0$ if $m \in\left\{m^{1}, m^{2}\right\}$ \\
\hline $\begin{array}{ll}m_{r^{\prime}+3}(t)=m^{3} & \text { if } \\
t \in\left[a^{1} / 4-25 / 2, a^{1} / 4+45 / 2\right) & \end{array}$ & $a_{r^{\prime}+3}(m)=t^{2} / 4-5 / 2$ if $m=m^{3}$ \\
\hline$m_{r^{\prime}+3}(t)=n^{1}$ if $t \in\left[a^{1} / 4+45 / 2,130\right]$ & $a_{r^{\prime}+3}(m)=70$ if $m=n^{1}$ \\
\hline
\end{tabular}

\begin{tabular}{|c|c|}
\hline Strategy Sender period $r^{\prime}+4$ & Strategy Receiver period $r^{\prime}+4$ \\
\hline $\begin{array}{ll}m_{r^{\prime}+4}(t) \sim U\left\{m^{1}, m^{2}\right\} & \text { if } \\
t \in\left[0, t^{2} / 8-5 / 4\right) & \end{array}$ & $a_{m+4}(m)=0$ if $m \in\left\{m^{1}, m^{2}, m^{3}\right\}$ \\
\hline $\begin{array}{ll}m_{r^{\prime}+4}(t)=m^{3} & \text { if } \\
t \in\left[t^{2} / 8-5 / 4, t^{2} / 8+135 / 4\right) & \end{array}$ & $a_{r^{\prime}+4}(m)=a^{1} / 8+225 / 4$ if $m=n^{1}$ \\
\hline$m_{r^{\prime}}+4(t)=n^{1}$ if $t \in\left[t^{2} / 8+135 / 4,130\right]$ & \\
\hline
\end{tabular}

Hence, starting at period $r^{\prime}$, we can characterize $f^{4}$ by $a_{p+1}^{1}=a_{p}^{1} / 8+225 / 4$, $t_{p}^{1}, t_{p+1}^{1}=t_{p}^{2} / 8-5 / 4$ and $t_{p+1}^{2}=135 / 4+t_{p+1}^{2} / 8\left(\right.$ as long as $0 \leq t_{p}^{1}<t_{p}^{2}<50$ and $\left.50<a_{p}^{1}<70\right)$.

$$
a_{p}^{1}=450 / 7, t_{p}^{2}=270 / 7 \text { and } t_{p}^{1}=25 / 7 \text { is a steady state and attractor to }
$$
which the dynamic converges monotonically. Hence, if in some period the strategy profile meets the conditions in $r^{\prime}$, then $f$ converges to the 4-cycle characterized by above values.

We proceed to give the first periods of the neologism dynamic for the babbling and naive initial conditions. We end as soon as the dynamic meets the sufficient conditions for their respective attractors specified above.

If we start with a babbling profile in period 1, the neologism dynamic proceeds as follows:

\begin{tabular}{|l|l|}
\hline Strategy Sender period 1 (Babbling) & Strategy Receiver period 1 (Babbling) \\
\hline$m_{1}(t) \sim U[0,130]$ if $t \in[0,130]$ & $a_{1}(m)=45$ for all $m \in[0,130]$ \\
\hline
\end{tabular}




\begin{tabular}{|l|l|}
\hline Strategy Sender period 2 (Babbling) & Strategy Receiver period 2 (Babbling) \\
\hline$m_{2}(t)=n^{1}$ if $t \in[0,45 / 2)$ & $a_{2}(m)=0$ if $m=n^{1}$ \\
\hline$m_{2}(t) \sim U[0,130] \quad$ if $t \in[45 / 2,115 / 2]$ & $a_{2}(m)=45$ if $m \in[0,130]$ \\
\hline$m_{2}(t)=n^{2}$ if $t \in(115 / 2,130]$ & $a_{2}(m)=70$ if $m=n^{2}$ \\
\hline
\end{tabular}

where $n^{1}=\langle 0,[0,45 / 2)\rangle$ and $n^{2}=\langle 70,(115 / 2,130]\rangle$.

\begin{tabular}{|l|l|}
\hline Strategy Sender period 3 (Babbling) & Strategy Receiver period 3 (Babbling) \\
\hline$m_{3}(t)=n^{1}$ if $t \in[0,45 / 2)$ & $a_{3}(m)=0$ if $m \in[0,130] \cup\left\{n^{1}\right\}$ \\
\hline$m_{3}(t) \sim U[0,130]$ if $t \in[45 / 2,115 / 2)$ & $a_{3}(m)=70$ if $m=n^{2}$ \\
\hline$m_{3}(t)=n^{2}$ if $t \in[115 / 2,130]$ & \\
\hline
\end{tabular}

\begin{tabular}{|l|l|}
\hline Strategy Sender period 4 (Babbling) & Strategy Receiver period 4 (Babbling) \\
\hline$m_{4}(t) \sim U[0,130] \cup n^{1}$ if $t \in[0,35)$ & $a_{4}(m)=0$ if $m \in[0,130] \cup n^{1}$ \\
\hline$m_{4}(t)=n^{2}$ if $t \in[35,130]$ & $a_{4}(m)=70$ if $m=n^{2}$ \\
\hline
\end{tabular}

\begin{tabular}{|l|l|}
\hline Strategy Sender period 5 (Babbling) & Strategy Receiver period 5 (Babbling) \\
\hline$m_{5}(t) \sim U[0,130] \cup n^{1} \quad$ if $t \in[0,35)$ & $a_{5}(m)=0$ if $m \in[0,130]$ cupn $^{1}$ \\
\hline$m_{5}(t)=n^{2}$ if $t \in[35,130]$ & $a_{5}(m)=125 / 2$ if $m=n^{2}$ \\
\hline
\end{tabular}

\begin{tabular}{|l|l|}
\hline Strategy Sender period 6 (Babbling) & Strategy Receiver period 6 (Babbling) \\
\hline$m_{6}(t) \sim U[0,130] \cup\left\{n^{1}\right\} \quad$ if $t \in[0,125 / 4)$ & $a_{6}(m)=0$ if $m \in[0,130] \cup\left\{n^{1}\right\}$ \\
\hline$m_{6}(t)=n^{2}$ if $t \in(125 / 4,265 / 4]$ & $a_{6}(m)=125 / 2$ if $m=n^{2}$ \\
\hline$m_{6}(t)=n^{3}$ if $t \in(265 / 4,130]$ & $a_{6}(m)=70$ if $m=n^{3}$ \\
\hline
\end{tabular}

where $n^{3}=\langle 70,(265 / 4,130]\rangle$.

\begin{tabular}{|l|l|}
\hline Strategy Sender period 7 (Babbling) & Strategy Receiver period 7 (Babbling) \\
\hline$m_{7}(t) \sim U[0,130] \cup\left\{n^{1}\right\} \quad$ if $t \in[0,125 / 4)$ & $a_{7}(m)=0$ if $m \in[0,130] \cup\left\{n^{1}\right\}$ \\
\hline$m_{7}(t)=n^{2}$ if $t \in[125 / 4,265 / 4)$ & $a_{7}(m)=25 / 4$ if $m=n^{2}$ \\
\hline$m_{7}(t)=n^{3}$ if $t \in[265 / 4,130]$ & $a_{7}(m)=70$ if $m=n^{3}$ \\
\hline
\end{tabular}

\begin{tabular}{|l|l|}
\hline Strategy Sender period 8 (Babbling) & Strategy Receiver period 8 (Babbling) \\
\hline$m_{8}(t) \sim U[0,130] \cup\left\{n^{1}\right\} \quad$ if $t \in[0,25 / 8)$ & $a_{8}(m)=0$ if $m \in[0,130] \cup\left\{n^{1}, n^{2}\right\}$ \\
\hline$m_{8}(t)=n^{2}$ if $t \in[25 / 8,305 / 8)$ & $a_{8}(m)=25 / 4$ if $m=n^{2}$ \\
\hline$m_{8}(t)=n^{3}$ if $t \in[305 / 8,130]$ & $a_{8}(m)=70$ if $m=n^{3}$ \\
\hline
\end{tabular}




\begin{tabular}{|c|c|}
\hline Strategy Sender period 9 (Babbling) & Strategy Receiver period 9 (Babbling) \\
\hline $\begin{array}{l}m_{9}(t) \sim U[0,130] \cup\left\{n^{1}, n^{2}\right\} \\
t \in[0,25 / 8)\end{array}$ & $a_{9}(m)=0$ if $m \in[0,130] \cup\left\{n^{1}, n^{2}\right\}$ \\
\hline$m_{9}(t)=n^{2}$ if $t \in[25 / 8,585 / 16]$ & $a_{9}(m)=1025 / 16$ if $m=n^{3}$ \\
\hline$m_{9}(t)=n^{3}$ if $t \in[585 / 16,130]$ & \\
\hline
\end{tabular}

\begin{tabular}{|c|c|}
\hline Strategy Sender period 10 (Babbling) & Strategy Receiver period 10 (Babbling) \\
\hline $\begin{array}{ll}m_{10}(t) \sim U[0,130] \cup\left\{n^{1}, n^{2}\right\} & \text { if } \\
t \in[0,1025 / 32) & \end{array}$ & $a_{10}(m)=0$ if $m \in[0,130] \cup\left\{n^{1}, n^{2}\right\}$ \\
\hline$m_{10}(t)=n^{3}$ if $t \in[1025 / 32,2145 / 32]$ & $a_{10}(m)=125 / 2$ if $m=n^{3}$ \\
\hline$m_{10}(t)=n^{4}$ if $t \in(2145 / 32,130]$ & $a_{10}(m)=70$ if $m=n^{4}$ \\
\hline
\end{tabular}

where $n^{4}=\langle 70,(2145 / 32,130]\rangle$.

\begin{tabular}{|c|c|}
\hline Strategy Sender period 11 (Babbling) & Strategy Receiver period 11 (Babbling) \\
\hline $\begin{array}{l}m_{11}(t) \sim U[0,130] \cup\left\{n^{1}, n^{2}\right\} \\
t \in[0,125 / 4)\end{array}$ & $a_{11}(m)=0$ if $m \in[0,130] \cup\left\{n^{1}, n^{2}\right\}$ \\
\hline$m_{11}(t)=n^{3}$ if $t \in[125 / 4,265 / 4)$ & $a_{11}(m)=225 / 32$ if $m=n^{3}$ \\
\hline$m_{11}(t)=n^{4}$ if $t \in[265 / 4,130]$ & $a_{11}(m)=70$ if $m=n^{4}$ \\
\hline
\end{tabular}

\begin{tabular}{|c|c|}
\hline Strategy Sender period 12 (Babbling) & Strategy Receiver period 12 (Babbling) \\
\hline 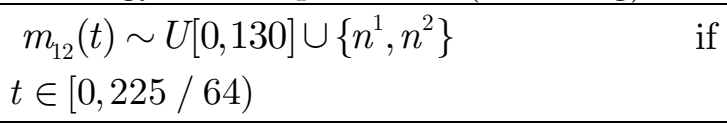 & $a_{12}(m)=0$ if $m \in[0,130] \cup\left\{n^{1}, n^{2}\right\}$ \\
\hline$m_{12}(t)=n^{3}$ if $t \in[225 / 64,2465 / 64)$ & $a_{12}(m)=25 / 4$ if $m=n^{3}$ \\
\hline$m_{12}(t)=n^{4}$ if $t \in[2465 / 64,130]$ & $a_{12}(m)=70$ if $m=n^{4}$ \\
\hline
\end{tabular}

\begin{tabular}{|c|c|}
\hline Strategy Sender period 13 (Babbling) & Strategy Receiver period 13 (Babbling) \\
\hline $\begin{array}{l}m_{13}(t) \sim U[0,130] \cup\left\{n^{1}, n^{2}\right\} \\
t \in[0,25 / 8)\end{array}$ & $a_{13}(m)=0$ if $m \in[0,130] \cup\left\{n^{1}, n^{2}, n^{3}\right\}$ \\
\hline$m_{13}(t)=n^{3}$ if $t \in[25 / 8,305 / 8)$ & $a_{13}(m)=8225 / 128$ if $m=n^{4}$ \\
\hline$m_{13}(t)=n^{4}$ if $t \in[305 / 8,130]$ & \\
\hline
\end{tabular}

Now, $t_{13}^{1}=25 / 8<t_{13}^{2}=305 / 8<50$ and $50<a_{13}^{1}=8225 / 128<70$. Hence, period 13 meets the requirements of round $r^{\prime}$ and the dynamic converges to the attracting four-cycle.

If we start with a naive profile in period 1, the neologism dynamic proceeds as follows: 


\begin{tabular}{|l|l|}
\hline Strategy Sender period 1 (Naive) & Strategy Receiver period 1 (Naive) \\
\hline$m_{1}(t)=t$ if $t \in[0,130]$ & $a_{1}(m)=0$ if $m \in[0,60]$ \\
\hline & $a_{1}(m)=m-60$ if $m \in[60,130]$ \\
\hline
\end{tabular}

\begin{tabular}{|l|l|}
\hline Strategy Sender period 2 (Naive) & Strategy Receiver period 2 (Naive) \\
\hline$m_{2}(t) \sim U[0,60]$ if $t=0$ & $a_{1}(m)=0$ if $m \in[0,60]$ \\
\hline$m_{2}(t)=t+60$ if $t \in(0,70)$ & $a_{1}(m)=m-60$ if $m \in[60,130]$ \\
\hline$m_{2}(t)=130$ if $t \in[70,130]$ & \\
\hline
\end{tabular}

\begin{tabular}{|l|l|}
\hline Strategy Sender period 3 (Naive) & Strategy Receiver period 3 (Naive) \\
\hline$m_{3}(t) \sim U[0,60]$ if $t=0$ & $a_{3}(m)=0$ if $m \in[0,120)$ \\
\hline$m_{3}(t)=t+60$ if $t \in[0,70)$ & $a_{3}(m)=m-120$ if $m \in[120,130)$ \\
\hline$m_{3}(t)=120$ if $t \in[70,130]$ & $a_{3}(m)=70$ if $m=130$ \\
\hline
\end{tabular}

\begin{tabular}{|l|l|}
\hline Strategy Sender period 4 (Naive) & Strategy Receiver period 4 (Naive) \\
\hline$m_{4}(t) \sim U[0,120]$ if $t=0$ & $a_{4}(m)=0$ if $m \in[0,120)$ \\
\hline$m_{4}(t)=t+120$ if $t \in[0,10)$ & $a_{4}(m)=m-120$ if $m \in[120,130)$ \\
\hline$m_{4}(t)=130-\epsilon$ if $t \in[10,40)$ & $a_{4}(m)=70$ if $m=130$ \\
\hline$m_{4}(t)=130$ if $t \in[40,130]$ & \\
\hline
\end{tabular}

\begin{tabular}{|l|l|}
\hline Strategy Sender period 5 (Naive) & Strategy Receiver period 5 (Naive) \\
\hline$m_{5}(t) \sim U[0,120]$ if $t=0$ & $a_{5}(m)=0$ if $m \in[0,130)$ \\
\hline$m_{5}(t)=t+120$ if $t \in[0,10)$ & $a_{5}(m)=65$ if $m=130$ \\
\hline$m_{5}(t)=130-\epsilon$ if $t \in[10,40)$ & \\
\hline$m_{5}(t)=130$ if $t \in[40,130]$ & \\
\hline
\end{tabular}

\begin{tabular}{|l|l|}
\hline Strategy Sender period 6 (Naive) & Strategy Receiver period 6 (Naive) \\
\hline$m_{6}(t) \sim U[0,130)$ if $t \in[0,65 / 2)$ & $a_{6}(m)=0$ if $m \in[0,130)$ \\
\hline$m_{6}(t)=130$ if $t \in[65 / 2,135 / 2]$ & $a_{6}(m)=65$ if $m=130$ \\
\hline$m_{6}(t)=n_{1}$ if $t \in(135 / 2,130]$ & $a_{6}(m)=70$ if $m=n_{1}$ \\
\hline
\end{tabular}

where $n_{1}=\langle 70,(135 / 2,130]\rangle$.

\begin{tabular}{|l|l|}
\hline Strategy Sender period 7 (Naive) & Strategy Receiver period 7 (Naive) \\
\hline$m_{7}(t) \sim U[0,130)$ if $t \in[0,65 / 2)$ & $a_{7}(m)=0$ if $m \in[0,130)$ \\
\hline$m_{7}(t)=130$ if $t \in[65 / 2,135 / 2]$ & $a_{7}(m)=15 / 2$ if $m=130$ \\
\hline$m_{7}(t)=n_{1}$ if $t \in(135 / 2,130]$ & $a_{7}(m)=70$ if $m=n_{1}$ \\
\hline
\end{tabular}




\begin{tabular}{|l|l|}
\hline Strategy Sender period 8 (Naive) & Strategy Receiver period 8 (Naive) \\
\hline$m_{8}(t) \sim U[0,130)$ if $t \in[0,15 / 4)$ & $a_{8}(m)=0$ if $m \in[0,130)$ \\
\hline$m_{8}(t)=130$ if $t \in[15 / 4,155 / 4]$ & $a_{8}(m)=15 / 2$ if $m=130$ \\
\hline$m_{8}(t)=n_{1}$ if $t \in(155 / 4,130]$ & $a_{8}(m)=70$ if $m=n_{1}$ \\
\hline
\end{tabular}

\begin{tabular}{|l|l|}
\hline Strategy Sender period 9 (Naive) & Strategy Receiver period 9 (Naive) \\
\hline$m_{9}(t) \sim U[0,130)$ if $\left.t \in[0,15 / 4)\right\}$ & $a_{9}(m)=0$ if $m \in[0,130]$ \\
\hline$m_{9}(t)=130$ if $\left.t \in[15 / 4,155 / 4]\right\}$ & $a_{9}(m)=515 / 8$ if $m=n_{1}$ \\
\hline$m_{9}(t)=n_{1}$ if $\left.t \in(155 / 4,130]\right\}$ & \\
\hline
\end{tabular}

Now, $t_{9}^{1}=15 / 4<t_{9}^{2}=155 / 4<50$ and $50<a_{9}^{1}=515 / 8<70$. Hence, period 9 meets the requirements of round $r^{\prime}$ and the dynamic converges to the attracting four-cycle.

Finally, we turn to the prediction errors for the attracting four-cycle. First the pooling equilibrium. In the same way as above, it can be straightforwardly calculated that prediction error of the pooling equilibrium in periods $r^{\prime}, r^{\prime}+1$, $r^{\prime}+2$ and $r^{\prime}+3$ is respectively equal to $\frac{17145}{637}, \frac{2585}{91}, \frac{304}{91}$ and $\frac{2640}{91}$. Hence, the average prediction error of the pooling equilibrium over the four cycle is $\frac{18750}{637} \simeq 29.4$. The prediction error of the separating equilibrium in periods $r^{\prime}$, $r^{\prime}+1, \quad r^{\prime}+2$ and $r^{\prime}+3$ is respectively equal to $\frac{4440}{637}, \frac{635}{91}, \frac{1825}{91}$ and $\frac{7625}{91}$. Hence, the average prediction error of the separating equilibrium over the four cycle is $\frac{29285}{2548} \simeq 11.5$.

\section{G(210)}

We continue with $B=210$. Consider the following state $r^{\prime}$ : 


\begin{tabular}{|l|l|}
\hline Strategy Sender period $r^{\prime}$ & Strategy Receiver period $r^{\prime}$ \\
\hline$m_{r^{\prime}}(t)=m^{1}$ if $t \in\left[0, t^{1}\right)$ & $a_{r^{\prime}}(m)=0$ if $m \in\left\{m^{1}, m^{2}\right\}$ \\
\hline$m_{r^{\prime}}(t)=m^{2}$ if $t \in\left[t^{1}, t^{2}\right)$ & $a_{r^{\prime}}(m)=a^{1}$ if $m=m^{3}$ \\
\hline$m_{r^{\prime}}(t)=m^{3}$ if $t \in\left[t^{2}, t^{3}\right)$ & $a_{r^{\prime}}(m)=a^{2}$ if $m=m^{4}$ \\
\hline$m_{r^{\prime}}(t)=m^{4}$ if $t \in\left[t^{3}, t^{4}\right)$ & $a_{r^{\prime}}(m)=a^{3}$ if $m=m^{5}$ \\
\hline$m_{r^{\prime}}(t)=m^{5}$ if $t \in\left[t^{4}, t^{5}\right]$ & $a_{r^{\prime}}(m)=a^{4}$ if $m=n^{1}$ \\
\hline$m_{r^{\prime}}(t)=n^{1}$ if $t \in\left(t^{5}, 210\right]$ & \\
\hline
\end{tabular}

$0<a^{1}<a^{2}<a^{3}<a^{4}$ with $a^{2}<30$ and $a^{4}<90$ and $n^{1}=\left\langle a^{4},\left[t^{5}, 210\right]\right\rangle$.

Then, by straightforwardly applying the neologism dynamic, we get for round $r^{\prime}+1$ :

\begin{tabular}{|c|c|}
\hline Strategy Sender period $r^{\prime}+1$ & Strategy Receiver period $r^{\prime}+1$ \\
\hline$m_{r^{\prime}+1}(t)=m^{1}$ if $t \in\left[0, a^{1} / 2\right)$ & $a_{r^{\prime}+1}(m)=0$ if $m \in\left\{m^{1}, m^{2}, m^{3}\right\}$ \\
\hline$m_{r^{\prime}+1}(t)=m^{3}$ if $t \in\left[a^{1} / 2,\left(a^{1}+a^{2}\right) / 2\right)$ & $a_{r^{\prime}+1}(m)=t^{4}-60$ if $m=m^{4}$ \\
\hline $\begin{array}{ll}m_{r^{\prime}+1}(t)=m^{4} & \text { if } \\
t \in\left[\left(a^{1}+a^{2}\right) / 2,\left(a^{2}+a^{3}\right) / 2\right) & \end{array}$ & $a_{r^{\prime}+1}(m)=t^{5}-60$ if $m=m^{5}$ \\
\hline $\begin{array}{ll}m_{r^{\prime}+1}(t)=m^{5} & \text { if } \\
t \in\left[\left(a^{2}+a^{3}\right) / 2,\left(a^{3}+a^{4}\right) / 2\right) & \end{array}$ & $a_{r^{\prime}+1}(m)=45+t^{5} / 2 \quad$ if $m=n^{1}$ \\
\hline $\begin{array}{l}m_{r^{\prime}+1}(t)=n^{1} \\
t \in\left[\left(a^{3}+a^{4}\right) / 2, \frac{2}{3}\left(45+a^{4}\right)\right]\end{array}$ & $a_{r^{\prime}+1}(m)=60+a^{4} / 3 \quad$ if $m=n^{2}$ \\
\hline$m_{r^{\prime}+1}(t)=n^{2}$ if $t \in\left(\frac{2}{3}\left(45+a^{4}\right), 210\right]$ & \\
\hline
\end{tabular}

where $n^{2}=\left\langle 60+a^{4} / 3,\left(\frac{2}{3}\left(45+a^{4}\right), 210\right]\right\rangle$.

Hence, for period $r \geq r^{\prime}$ we can describe $f$ by $a_{r+1}^{4}=60+a_{r}^{4} / 3$, $t_{r+1}^{5}=\frac{2}{3}\left(45+a_{r}^{4} / 3\right), \quad a_{r+1}^{3}=45+t_{r}^{5} / 2, \quad t_{r+1}^{4}=\frac{1}{2}\left(a_{r}^{3}+a_{r}^{4}\right), \quad a_{r+1}^{2}=t_{r}^{5}-60$, $t_{r+1}^{3}=\frac{1}{2}\left(a_{r}^{2}+a_{r}^{3}\right), \quad a_{r+1}^{1}=t_{r}^{4}-60, t_{r+1}^{2}=\frac{1}{2}\left(a_{r}^{1}+a_{r}^{2}\right)$ and $t_{r+1}^{1}=\frac{1}{2} a_{r}^{1} \quad$ as long as $a_{r}^{1}, \ldots, a_{r}^{5}$ and $t_{r}^{1}, \ldots, t_{r}^{5}$ meet the above conditions).

Since $a_{r+1}^{4}=60+a_{r}^{4} / 3, a_{r}^{4}$ converges monotonically to 90 . Consequently, it follows that 


$$
a_{r}^{4}=90, t_{r}^{5}=90, \quad a_{r}^{3}=90, \quad t_{r}^{4}=90, \quad a_{r}^{2}=30, \quad t_{r}^{3}=60, a_{r}^{1}=30, \quad t_{r}^{2}=30
$$

and $t_{r}^{1}=15$ is an attractor for this dynamic to which converges. (It is not a steady state, as if $a_{r}^{4}=90$, then no neologism could be made. Nonetheless, the profile is never reached and all points in its neighborhood converge to it.)

We now proceed to give the first periods of the neologism dynamic for the babbling and naive initial conditions.

If we start with a babbling profile in period 1, the neologism dynamic proceeds as follows:

\begin{tabular}{|l|l|}
\hline Strategy Sender period 1 (Babbling) & Strategy Receiver period 1 (Babbling) \\
\hline$m_{1}(t) \sim U[0,210]$ if $t \in[0,210]$ & $a_{1}(m)=45$ for all $m \in[0,210]$ \\
\hline
\end{tabular}

\begin{tabular}{|l|l|}
\hline Strategy Sender period 2 (Babbling) & Strategy Receiver period 2 (Babbling) \\
\hline$m_{2}(t)=n^{1}$ if $t \in[0,45 / 2)$ & $a_{2}(m)=0$ if $m=n^{1}$ \\
\hline$m_{2}(t) \sim U[0,210]$ if $t \in[45 / 2,60]$ & $a_{2}(m)=45$ if $m \in[0,210]$ \\
\hline$m_{2}(t)=n^{2}$ if $t \in(60,210]$ & $a_{2}(m)=75$ if $m=n^{2}$ \\
\hline
\end{tabular}

where $n^{1}=\langle 0,[0,45 / 2)\rangle$ and $n^{2}=\langle 75,(60,210]\rangle$.

\begin{tabular}{|l|l|}
\hline Strategy Sender period 3 (Babbling) & Strategy Receiver period 3 (Babbling) \\
\hline$m_{3}(t)=n^{1}$ if $t \in[0,45 / 2)$ & $a_{3}(m)=0$ if $m \in[0,210] \cup\left\{n^{1}\right\}$ \\
\hline$m_{3}(t) \sim U[0,210]$ if $t \in[45 / 2,60)$ & $a_{3}(m)=75$ if $m=n^{2}$ \\
\hline$m_{3}(t)=n^{2}$ if $t \in[60,80]$ & $a_{3}(m)=85$ if $m=n^{3}$ \\
\hline$m_{3}(t)=n^{3}$ if $t \in(80,210]$ & \\
\hline
\end{tabular}

where $n^{3}=\langle 85,(80,210]\rangle$.

\begin{tabular}{|l|l|}
\hline Strategy Sender period 4 (Babbling) & Strategy Receiver period 4 (Babbling) \\
\hline$m_{4}(t) \sim U[0,210] \cup\left\{n^{1}\right\}$ if $t \in[0,75 / 2)$ & $a_{4}(m)=0$ if $m \in[0,210] \cup\left\{n^{1}\right\}$ \\
\hline$m_{4}(t)=n^{2}$ if $t \in[75 / 2,80)$ & $a_{4}(m)=20$ if $m=n^{2}$ \\
\hline$m_{4}(t)=n^{3}$ if $t \in[80,260 / 3]$ & $a_{4}(m)=85$ if $m=n^{3}$ \\
\hline$m_{4}(t)=n^{4}$ if $t \in(260 / 3,210]$ & $a_{4}(m)=265 / 3$ if $m=n^{4}$ \\
\hline
\end{tabular}

where $n^{4}=\langle 265 / 3,(260 / 3,210]\rangle$. 


\begin{tabular}{|l|l|}
\hline Strategy Sender period 5 (Babbling) & Strategy Receiver period 5 (Babbling) \\
\hline$m_{5}(t) \sim U[0,210] \cup\left\{n^{1}\right\}$ if $t \in[0,10)$ & $a_{5}(m)=0$ if $m \in[0,210] \cup\left\{n^{1}\right\}$ \\
\hline$m_{5}(t)=n^{2}$ if $t \in[10,105 / 2)$ & $a_{5}(m)=20$ if $m=n^{2}$ \\
\hline$m_{5}(t)=n^{3}$ if $t \in[105 / 2,260 / 3)$ & $a_{5}(m)=80 / 3$ if $m=n^{3}$ \\
\hline$m_{5}(t)=n^{4}$ if $t \in[260 / 3,800 / 9]$ & $a_{5}(m)=265 / 3$ if $m=n^{4}$ \\
\hline$m_{5}(t)=n^{5}$ if $t \in(800 / 9,210]$ & $a_{5}(m)=805 / 9$ if $m=n^{5}$ \\
\hline
\end{tabular}

where $n^{5}=\langle 805 / 9,(800 / 9,210]\rangle$.

\begin{tabular}{|l|l|}
\hline Strategy Sender period 6 (Babbling) & Strategy Receiver period 6 (Babbling) \\
\hline$m_{6}(t) \sim U[0,210] \cup\left\{n^{1}\right\}$ if $t \in[0,10)$ & $a_{6}(m)=0$ if $m \in[0,210] \cup\left\{n^{1}, n^{2}\right\}$ \\
\hline$m_{6}(t)=n^{2}$ if $t \in[10,70 / 3)$ & $a_{6}(m)=80 / 3$ if $m=n^{3}$ \\
\hline$m_{6}(t)=n^{3}$ if $t \in[70 / 3,115 / 2)$ & $a_{6}(m)=260 / 9$ if $m=n^{4}$ \\
\hline$m_{6}(t)=n^{4}$ if $t \in[115 / 2,800 / 9)$ & $a_{6}(m)=805 / 9$ if $m=n^{5}$ \\
\hline$m_{6}(t)=n^{5}$ if $t \in[800 / 9,2420 / 5]$ & $a_{6}(m)=2425 / 27$ if $m=n^{6}$ \\
\hline$m_{6}(t)=n^{6}$ if $t \in(2420 / 27,210]$ & \\
\hline
\end{tabular}

where $n^{6}=\langle 2425 / 27,(2420 / 27,210]\rangle$.

$$
\text { Now, } 0<t_{6}^{1}=10<15, t_{6}^{3}=115 / 2<60, t_{6}^{5}=2420 / 27<90, a_{6}^{2}=260 / 9<30
$$

and $a_{6}^{4}=2425 / 27<90 \quad$ Hence, period 6 meets the requirements of round $r^{\prime}$ and the dynamic converges to the attractor.

If we start with a naive profile in period 1, the neologism dynamic proceeds as follows:

\begin{tabular}{|l|l|}
\hline Strategy Sender period 1 (Naive) & Strategy Receiver period 1 (Naive) \\
\hline$m_{1}(t)=t \quad$ if $t \in[0,210]$ & $a_{1}(m)=0$ if $m \in[0,60]$ \\
\hline & $a_{1}(m)=m-60$ if $m \in[60,210]$ \\
\hline
\end{tabular}

\begin{tabular}{|l|l|}
\hline Strategy Sender period 2 (Naive) & Strategy Receiver period 2 (Naive) \\
\hline$m_{2}(t) \sim U[0,60]$ if $t=0$ & $a_{2}(m)=0$ for all $m \in[0,60]$ \\
\hline$m_{2}(t)=t+60$ if $t \in(0,150)$ & $a_{2}(m)=m-60$ for all $m \in[60,210]$ \\
\hline$m_{2}(t)=210$ if $t \in[150,210]$ & \\
\hline
\end{tabular}




\begin{tabular}{|l|l|}
\hline Strategy Sender period 3 (Naive) & Strategy Receiver period 3 (Naive) \\
\hline$m_{3}(t) \sim U[0,60]$ if $t=0$ & $a_{3}(m)=0$ if $m \in[0,120)$ \\
\hline$m_{3}(t)=t+60$ if $t \in(0,150)$ & $a_{3}(m)=m-120$ if $m \in[120,210)$ \\
\hline$m_{3}(t)=210$ if $t \in[150,210]$ & $a_{3}(m)=120$ if $m=210$ \\
\hline
\end{tabular}

\begin{tabular}{|l|l|}
\hline Strategy Sender period 4 (Naive) & Strategy Receiver period 4 (Naive) \\
\hline$m_{4}(t) \sim U[0,120]$ if $t=0$ & $a_{4}(m)=0$ if $m \in[0,120)$ \\
\hline$m_{4}(t)=t+120$ if $t \in(0,90)$ & $a_{4}(m)=m-120$ if $m \in[120,210)$ \\
\hline$m_{4}(t)=210-\epsilon$ if $t \in[90,105)$ & $a_{4}(m)=120$ if $m=210$ \\
\hline$m_{4}(t)=210$ if $t \in[105,210]$ & \\
\hline
\end{tabular}

\begin{tabular}{|l|l|}
\hline Strategy Sender period 5 (Naive) & Strategy Receiver period 5 (Naive) \\
\hline$m_{5}(t) \sim U[0,120]$ if $t=0$ & $a_{5}(m)=0$ if $m \in[0,180)$ \\
\hline$m_{5}(t)=t+120$ if $t \in(0,90)$ & $a_{5}(m)=m-180$ if $m \in[180,210-\epsilon)$ \\
\hline$m_{5}(t)=210-\epsilon$ if $t \in[90,105)$ & $a_{5}(m)=45$ if $m=210-\epsilon$ \\
\hline$m_{5}(t)=210$ if $t \in[105,210]$ & $a_{5}(m)=195 / 2$ if $m=210$ \\
\hline
\end{tabular}

\begin{tabular}{|l|l|}
\hline Strategy Sender period 6 (Naive) & Strategy Receiver period 6 (Naive) \\
\hline$m_{6}(t) \sim U[0,180]$ if $t=0$ & $a_{6}(m)=0$ if $m \in[0,180)$ \\
\hline$m_{6}(t)=t+180$ if $t \in(0,30)$ & $a_{6}(m)=m-180$ if $m \in[180,210-\epsilon)$ \\
\hline$m_{6}(t)=210-2 \epsilon$ if $t \in[30,75 / 2)$ & $a_{6}(m)=45$ if $m=210-\epsilon$ \\
\hline$m_{6}(t)=210-\epsilon$ if $t \in[75 / 2,285 / 4)$ & $a_{6}(m)=195 / 2$ if $m=210$ \\
\hline$m_{6}(t)=210$ if $t \in[285 / 4,210]$ & \\
\hline
\end{tabular}

\begin{tabular}{|l|l|}
\hline Strategy Sender period 7 (Naive) & Strategy Receiver period 7 (Naive) \\
\hline$m_{6}(t) \sim U[0,180]$ if $t=0$ & $a_{7}(m)=0$ if $m \in[0,210-2 \epsilon)$ \\
\hline$m_{7}(t)=t+180$ if $t \in(0,30)$ & $a_{7}(m)=45 / 4$ if $m=210-\epsilon$ \\
\hline$m_{7}(t)=210-2 \epsilon$ if $t \in[30,75 / 2)$ & $a_{7}(m)=645 / 8$ if $m=210$ \\
\hline$m_{7}(t)=210-\epsilon$ if $t \in[75 / 2,285 / 4)$ & \\
\hline$m_{7}(t)=210$ if $t \in[285 / 4,210]$ & \\
\hline
\end{tabular}

\begin{tabular}{|l|l|}
\hline Strategy Sender period 8 (Naive) & Strategy Receiver period 8 (Naive) \\
\hline$m_{8}(t) \sim U[0,210-2 \epsilon)$ if $t \in[0,45 / 8)$ & $a_{8}(m)=0$ if $m \in[180,210-2 \epsilon)$ \\
\hline$m_{8}(t)=210-\epsilon$ if $t \in[45 / 8,735 / 16)$ & $a_{8}(m)=45 / 4$ if $m=210-\epsilon$ \\
\hline$m_{8}(t)=210$ if $t \in[735 / 16,335 / 4)$ & $a_{8}(m)=645 / 8$ if $m=210$ \\
\hline$m_{8}(t)=n^{1}$ if $t \in[335 / 4,210]$ & $a_{8}(m)=695 / 8$ if $m=n^{1}$ \\
\hline
\end{tabular}

where $n^{1}=\langle 695 / 8,(335 / 4,210]\rangle$. 


\begin{tabular}{|l|l|}
\hline Strategy Sender period 9 (Naive) & Strategy Receiver period 9 (Naive) \\
\hline$m_{9}(t) \sim U[0,210-2 \epsilon)$ if $t \in[0,45 / 8)$ & $a_{9}(m)=0$ if $m \in[0,210-\epsilon)$ \\
\hline$m_{9}(t)=210-\epsilon$ if $t \in[45 / 8,735 / 16)$ & $a_{9}(m)=95 / 4$ if $m=210$ \\
\hline$m_{9}(t)=210$ if $t \in[735 / 16,335 / 4)$ & $a_{9}(m)=695 / 8$ if $\mathrm{f} m=n^{1}$ \\
\hline$m_{9}(t)=n^{1}$ if $t \in[335 / 4,1055 / 12]$ & $a_{9}(m)=2135 / 24$ if $m=n^{2}$ \\
\hline$m_{9}(t)=n^{2}$ if $t \in(1055 / 12,210]$ & \\
\hline
\end{tabular}

where $n^{2}=\langle 2135 / 24,(1055 / 12,210]\rangle$.

\begin{tabular}{|l|l|}
\hline Strategy Sender period 10 (Naive) & Strategy Receiver period 10 (Naive) \\
\hline$m_{10}(t) \sim U[0,210-\epsilon)$ if $t \in[0,95 / 8)$ & $a_{10}(m)=0$ if $m \in[0,210-\epsilon)$ \\
\hline$m_{10}(t)=210$ if $t \in[95 / 8,885 / 16)$ & $a_{10}(m)=95 / 4$ if $m=210$ \\
\hline$m_{10}(t)=n^{1}$ if $t \in[885 / 16,1055 / 12)$ & $a_{10}(m)=335 / 12$ if $m=n^{1}$ \\
\hline$m_{10}(t)=n^{2}$ if $t \in[1055 / 12,3215 / 36]$ & $a_{10}(m)=2135 / 24$ if $m=n^{2}$ \\
\hline$m_{10}(t)=n^{3}$ if $t \in(3215 / 36,210]$ & $a_{10}(m)=6455 / 72$ if $m=n^{3}$ \\
\hline
\end{tabular}

where $n^{3}=\langle 6455 / 72,(3215 / 36,210]\rangle$.

\begin{tabular}{|l|l|}
\hline Strategy Sender period 11 (Naive) & Strategy Receiver period 11 (Naive) \\
\hline$m_{11}(t) \sim U[0,210-\epsilon)$ if $t \in[0,95 / 8)$ & $a_{11}(m)=0$ if $m \in[0,210]$ \\
\hline$m_{11}(t)=210$ if $t \in[95 / 8,155 / 6)$ & $a_{11}(m)=335 / 12$ if $m=n^{1}$ \\
\hline$m_{11}(t)=n^{1}$ if $t \in[155 / 6,935 / 16)$ & $a_{11}(m)=1055 / 36$ if $m=n^{2}$ \\
\hline$m_{11}(t)=n^{2}$ if $t \in[935 / 16,3215 / 36)$ & $a_{11}(m)=6455 / 72$ if $m=n^{3}$ \\
\hline$m_{11}(t)=n^{3}$ if $t \in[3215 / 36,9695 / 108]$ & $a_{11}(m)=19415 / 216$ if $m=n^{4}$ \\
\hline$m_{11}(t)=n^{4}$ if $t \in(9695 / 108,210]$ & \\
\hline
\end{tabular}

where $n^{4}=\langle 19415 / 216,(9695 / 108,210]\rangle$.

Now, $\quad t_{11}^{1}=95 / 8<15, \quad t_{11}^{3}=935 / 16<60, \quad t_{11}^{5}=9695 / 108<90$, $a_{11}^{2}=1055 / 36<30$ and $a_{11}^{4}=19415 / 216<90$. Hence, period 11 meets the requirements of round $r^{\prime}$ and the dynamic converges to the attractor.

Finally, we turn to the prediction errors for of the equilibria with respect to the attractor. The average prediction error of the pooling equilibrium is equal to $\frac{285}{7} \simeq 40.7$. The average prediction error of the separating equilibrium is equal to $\frac{195}{7} \simeq 27.9$ 


\section{Appendix C (Online): Instructions}

We include the experimental instructions (including check questions) of the G(120) treatment for both the "Chooser" (Sender) and "Proposer" (Receiver) roles. The instructions of the $\mathrm{G}(130)$ and $\mathrm{G}(210)$ treatments are very similar. 


\section{FOR ONLINE PUBLICATION}

Instructions Chooser

\section{INSTRUCTIONS}

Welcome to this decision-making experiment. Please read these instructions carefully. We will first provide you with an outline of the instructions and then we will proceed with a detailed description of the instructions.

\section{OUTLINE}

\section{Experiment}

- At the start of the experiment you will receive a starting capital of 100 points. In addition, you can earn points with your decisions.

- At the end of the experiment, you receive 1,5 (one-and-a-half) euro for each 100 points earned.

- $\quad$ The experiment consists of around 50 periods.

- Your role in the whole experiment is: CHOOSER.

- In each period, you will be randomly paired with a different participant who performs the role of Proposer.

\section{Sequence of events}

- In each period, you and the Proposer will bargain over an outcome, which can be any number between 0 and 120 .

- Your preferred outcome is a number between 0 and 120 . Any number between 0 and 120 is equally likely. The Proposer's preferred outcome is always 0 .

- Each period you will receive a new (random) preferred outcome. You are the only one who is informed about your preferred outcome.

- After learning your preferred outcome, you will send a SUGGESTION for a proposal (between 0 and 120) to the Proposer.

- The Proposer is informed of your suggestion and makes a PROPOSAL (between 0 and 120) for the outcome.

- After you have been informed of the proposal, you accept or reject it.

- At the end of a period, you are informed of the points you earned (your payoff).

\section{Payoffs}

- When you accept a proposal, your payoff is 60 minus the distance between your preferred outcome and the proposal.

- The Proposer's payoff is 60 minus 0.4 times the proposal in this case.

- When you reject a proposal, you receive 0 points and the Proposer receives 0 points.

\section{History Overview}

When making a decision, you may use the History Overview, which provides an overview of the results of the other Chooser/Proposer pairs (including your own pair) in the 15 most recent periods. The left part of the overview is a Table with four columns SUGGESTION, PROPOSAL, ACCEPTANCE and PREFERRED OUTCOME. In a row, you will find a particular pair's suggestion, the corresponding proposal, whether the Chooser accepted or rejected the proposal and the preferred outcome of that Chooser. On the right, you find a Graph where the most recent results are represented by blue squares. On the horizontal axis you can read the value of the suggestion and on the vertical axis the value of the corresponding proposal 


\section{FOR ONLINE PUBLICATION}

\section{DETAILED INSTRUCTIONS}

Now we will describe the experiment in detail. At the start of the experiment you will receive a starting capital of 100 points. During the experiment you will be asked to make a number of decisions. Your decisions and the decisions of the participants you will be paired with will determine how much money you earn. The experiment consists of around 50 periods. In each period, your earnings will be denoted in points. Your final earnings in the experiment will be equal to the starting capital plus the sum of your earnings in all periods. At the end of the experiment, your earnings in points will be transferred to money. For each 100 points you earn, you will receive 1,5 (one-and-a-half) euro. Your earnings will be privately paid to you in cash. In each period, all participants are paired in couples. One participant within a pair has the role of CHOOSER, the other participant performs the role of PROPOSER. In all periods you keep the same role.

Your role is: CHOOSER.

\section{MATCHING PROCEDURE}

For the duration of the experiment, you will be in a fixed matching group of five Proposers and five Choosers (hence 10 participants in total, including yourself). In each period you are randomly matched to another participant in this matching group with the role of Proposer. You will never learn with whom you are matched.

\section{BARGAINING AND PREFERRED OUTCOMES}

In each period, you and the Proposer with whom you are coupled will bargain over an outcome. The Proposer's preferred outcome is always 0. Your preferred outcome is a number between (and including) 0 and 120. Any number between 0 and 120 is equally likely. Each period you will receive a new preferred outcome that does not depend on your preferred outcome of any previous period. You are the only one who is informed about your preferred outcome. The Proposer only knows that your preferred outcome is a number between 0 and 120 (and that each such number is equally likely).

\section{SEQUENCE OF EVENTS IN A PERIOD}

After you have learned your preferred outcome in a period, you will send a SUGGESTION for a proposal to the Proposer. You may send any suggestion between (and including) 0 and 120. It is up to you to decide whether and how you let your suggestion depend on your preferred outcome. Then, the Proposer with whom you are coupled is informed of your suggestion (but not of your preferred outcome). Subsequently, the Proposer makes a PROPOSAL for the outcome. A proposal is any number between (and including) 0 and 120. Finally, you will choose to accept or reject the proposal.

At the end of a period, you are informed of the payoff (points you earned) that you made. This payoff is automatically added to your total earnings (or in case that you make a loss, it is subtracted from your total earnings). The Proposer is informed of the outcome, your preferred outcome and her or his own payoff.

Please note that the experiment will only continue from one phase to another after everybody has pressed OK/PROCEED. For this reason, please press OK/PROCEED as soon as you have made your decision. 


\section{PAYOFFS WHEN YOU ACCEPT THE PROPOSAL}

When you accept the proposal, you will receive a payoff of 60 minus the distance between your preferred outcome and the proposal:

Your payoff $=60-$ distance(your preferred outcome and proposal).

When you accept the proposal, the Proposer's payoff is 60 minus 0.4 times the proposal:

Payoff Proposer $=60-0.4 *$ proposal.

It is possible to reject a proposal.

\section{PAYOFFS WHEN YOU REJECT THE PROPOSAL}

When you reject a proposal, then the outcome is the status quo. In this case, you will receive 0 points and the Proposer will receive 0 points.

Notice that accepting an offer gives you a higher payoff than rejecting it if and only if the distance between the proposal and your preferred outcome is smaller than 60. The Proposer's payoff is higher when you accept than when you reject in all cases.

EXAMPLE 1. Suppose your preferred outcome is 80 and you receive a proposal of 100 . Then, the distance between your preferred outcome and proposal is $100-80=20$.

If you accept, your payoff is $60-20=40$. The Proposer's payoff in this case is $60-0.4 * 100=$ 20.

If you reject, your payoff is 0 and the Proposer's payoff is 0 .

EXAMPLE 2. Suppose your preferred outcome is 80 and you receive a proposal of 10 . Then, the distance between your preferred outcome and the proposal is $80-10=70$.

If you accept, your payoff is $60-70=-10$. The Proposer's payoff in this case is $60-0.4 * 10$ $=56$.

If you reject, your payoff is 0 and the Proposer's payoff is 0 .

\section{HISTORY OVERVIEW}

When making a decision, you may use the History Overview, which fills the lower part of the screen. The History Overview summarizes the results of the most recent 15 periods. (If less than 15 periods have been completed, this history overview contains results of all completed periods.) Apart from your own results in the previous periods, the History Overview also contains the results of the other Chooser/Proposer pairs in your matching group. In total you are thus informed about the past results of the same matching group of five Chooser/Proposer pairs. All other Choosers and Proposers in your matching group will have the same information. The presentation of information for Proposers is different than for Choosers.

\section{TABLE}

Below you see an example of the history overview. THE NUMBERS IN THE HISTORY OVERVIEW DO NOT INDICATE WHAT YOU SHOULD DO IN THE EXPERIMENT. The left part of the history overview is a Table with four columns. The first column labelled SUGGESTION contains the suggestions made by the Choosers in the recent previous periods. The second column labelled PROPOSAL gives the proposal that was made by the Proposer as a response to the suggestion in the same row. The third column labelled ACCEPTANCE shows whether the Chooser accepted or rejected the proposal. The fourth column labelled PREFERRED OUTCOME shows the preferred outcome of the Chooser. 


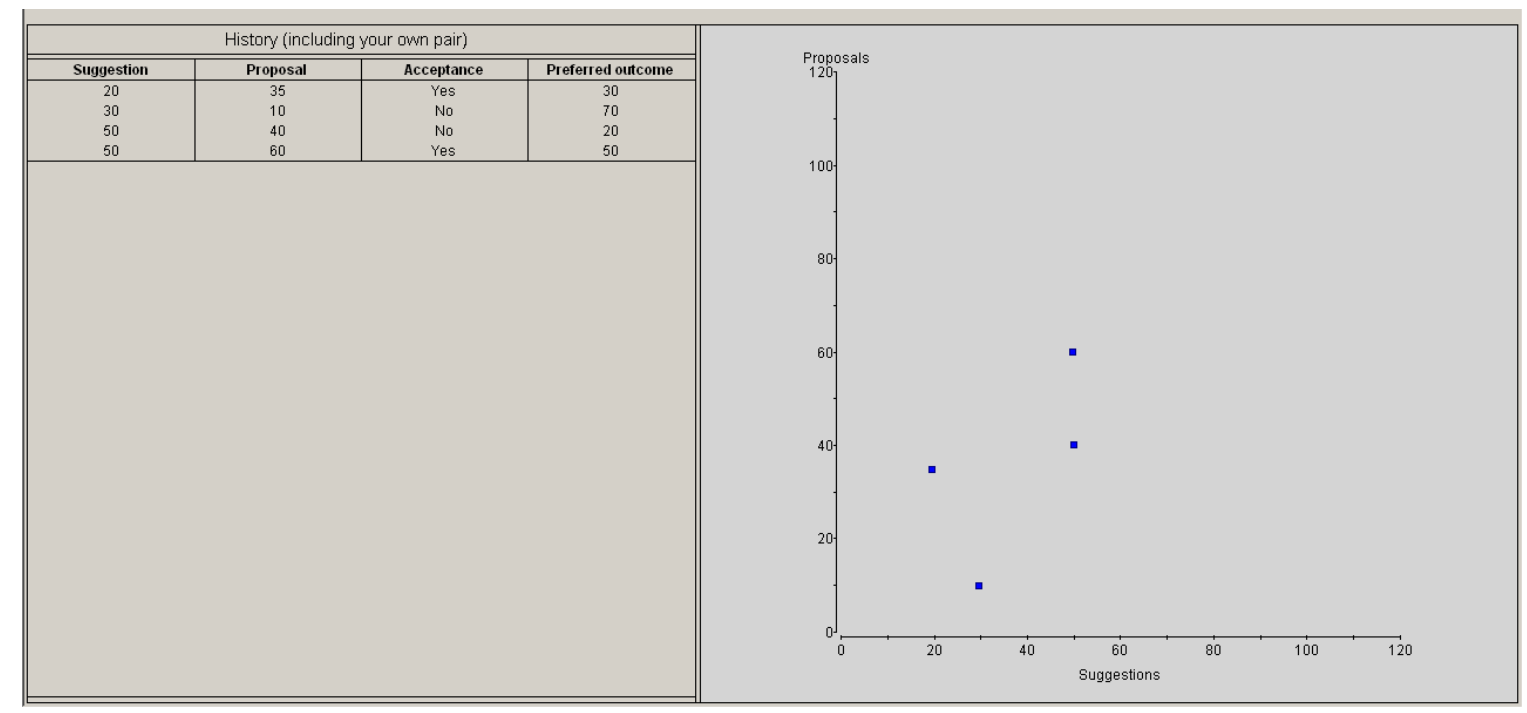

The results shown in the history overview will be sorted on the basis of suggestion in ascending order. (The lower the suggestion, the higher the place in the table.) When the suggestion is the same for two or more different results, these observations will be sorted on the basis of proposal, again in ascending order. In the example above, this applies to the third and the fourth row, where two Choosers chose the same suggestion but the corresponding Proposers chose different proposals. More generally, observations have been sorted first on suggestion, then on proposal, then on acceptance or rejection and finally on preferred outcome.

\section{GRAPH}

On the right of the history overview, the most recent results are represented in a graph. The horizontal axis presents the suggestion and the vertical axis presents the proposal. Each previous observation is represented by a blue square. On the horizontal axis you can read the value of the suggestion for a particular result and on the vertical axis you can read the value of the corresponding proposal. (Proposers will see preferred outcomes on the vertical axis, rather than proposals.)

EXAMPLE. Consider the square that is displayed in the lower left corner of the Graph shown above. Here, the Chooser made a suggestion of 30. The Proposer responded with a proposal of 10.

You have now reached the end of the instructions. The next page contains some questions concerning the experiment. When all participants have answered all questions correctly, we will proceed with the experiment. 


\section{QUESTIONS}

Please answer the following questions. THE VALUES USED IN SOME QUESTIONS DO NOT INDICATE WHAT YOU SHOULD DO IN THE EXPERIMENT. RATHER, THEY HAVE BEEN CHOSEN TO FACILITATE CALCULATIONS.

1. Is the following statement correct? 'In each period I am coupled with the same Proposer.'

2. Is the following statement correct? 'My preferred position will be observed by the Proposer before (s)he makes her or his proposal.'

3.

(A) What is the highest value your preferred outcome can take on?

(B) What is the highest value a suggestion of yours can take on?

(C) What is the highest value a proposal can take on?

4. Consider a period in which your preferred outcome is 50 . You chose to send a suggestion of 40. The Proposer made a proposal of 20, which was accepted by you.

(A) What are your own earnings in this period?

(B) How much does the Proposer to whom you are paired earn?

5. Consider a period in which your preferred outcome is 90 . You chose to send a suggestion of 100. The Proposer made a proposal of 0 , which was accepted by you.

(A) What are your own earnings in this period?

(B) How much does the Proposer to whom you are paired earn?

6. Consider a period in which your preferred outcome is 30. You chose to send a suggestion of 40. The Proposer made a proposal of 10, which was rejected by you.

(A) What are your own earnings in this period?

(B) How much does the Proposer to whom you are paired earn?

When you are ready answering the questions, please raise your hand. 
Instructions Proposer

\section{INSTRUCTIONS}

Welcome to this decision-making experiment. Please read these instructions carefully. We will first provide you with an outline of the instructions and then we will proceed with a detailed description of the instructions.

\section{OUTLINE}

\section{Experiment}

- At the start of the experiment you will receive a starting capital of 100 points. In addition, you can earn points with your decisions.

- At the end of the experiment, you receive 1,5 (one-and-a-half) euro for each 100 points earned.

- $\quad$ The experiment consists of around 50 periods.

- Your role in the whole experiment is: PROPOSER.

- In each period, you will be randomly paired with a different participant who performs the role of Chooser.

\section{Sequence of events}

- In each period, you and the Chooser will bargain over an outcome, which can be any number between 0 and 120 .

- $\quad$ Your preferred outcome is always 0.

- The Chooser's preferred outcome is a number between 0 and 120 . Any number between 0 and 120 is equally likely.

- $\quad$ Each period, each Chooser will receive a new (random) preferred outcome. The Chooser is the only one who is informed about her or his preferred outcome.

- $\quad$ After learning her or his preferred outcome, the Chooser with whom you are matched will send a SUGGESTION for a proposal (between 0 and 120) to you.

- You are informed of the Chooser's suggestion and make a PROPOSAL (between 0 and 120) for the outcome.

- After the Chooser has been informed of the proposal, she or he accepts or rejects it.

- $\quad$ At the end of a period, you are informed of the points you earned (your payoff).

\section{Payoffs}

- When the Chooser accepts your proposal, your payoff is 60 minus 0.4 times the proposal.

- The Chooser's payoff is in this case 60 minus the distance between her or his preferred outcome and the proposal.

- $\quad$ When the Chooser rejects your proposal, you receive 0 points and the Chooser 0 points.

\section{History Overview}

When making a decision, you may use the History Overview, which provides an overview of the results of five Chooser/Proposer pairs (including your own pair) in the 15 most recent periods. The left part of the overview is a Table with four columns SUGGESTION, PREFERRED OUTCOME, PROPOSAL and ACCEPTANCE. In a row, you will find a particular pair's suggestion, the preferred outcome of the Chooser, the proposal made by the Proposer and whether the Chooser accepted or rejected the proposal. On the right, you find a Graph where the most recent results are represented by blue squares. On the horizontal axis you can read the value of the suggestion and on the vertical axis the value of the corresponding preferred outcome of the Chooser. 


\section{DETAILED INSTRUCTIONS}

Now we will describe the experiment in detail. At the start of the experiment you will receive a starting capital of 100 points. During the experiment you will be asked to make a number of decisions. Your decisions and the decisions of the participants you will be paired with will determine how much money you earn. The experiment consists of around 50 periods. In each period, your earnings will be denoted in points. Your final earnings in the experiment will be equal to the starting capital plus the sum of your earnings in all periods. At the end of the experiment, your earnings in points will be transferred to money. For each 100 points you earn, you will receive 1,5 (one-and-a-half) euro. Your earnings will be privately paid to you in cash. In each period, all participants are paired in couples. One participant within a pair has the role of CHOOSER, the other participant performs the role of PROPOSER. In all periods you keep the same role.

Your role is: PROPOSER.

\section{MATCHING PROCEDURE}

For the duration of the experiment, you will be in a fixed matching group of five Proposers and five Choosers (hence 10 participants in total, including yourself). In each period you are randomly matched to another participant with the role of Chooser. You will never learn with whom you are matched.

\section{BARGAINING AND PREFERRED OUTCOMES}

In each period, you and the Chooser with whom you are coupled will bargain over an outcome. Your preferred outcome is always 0. The Chooser's preferred outcome is a number between (and including) 0 and 120. Any number between 0 and 120 is equally likely. Each period, each Chooser will receive a new preferred outcome that does not depend on a preferred outcome of any previous period. The Chooser is the only one who is informed about her or his preferred outcome. You only know that the Chooser's preferred outcome is a number between 0 and 120 (and that each such number is equally likely).

\section{SEQUENCE OF EVENTS IN A PERIOD}

After the Chooser with whom you are matched has learned her or his preferred outcome in a period, she or he will send a SUGGESTION for a proposal to you. The Chooser may send any suggestion between (and including) 0 and 120. It is up to the Chooser to decide whether and how she or he lets her or his suggestion depend on her or his preferred outcome. Then, you are informed of the Chooser's suggestion (but not of her or his preferred outcome). Subsequently, you make a PROPOSAL for the outcome. A proposal is any number between (and including) 0 and 120. Finally, the Chooser will choose to accept or reject the proposal.

At the end of a period, you are informed of the outcome of the period and the preferred outcome of the Chooser you were paired with. Finally, you are informed of the payoff (points you earned) that you made. This payoff is automatically added to your total earnings (or in case that you make a loss, it is subtracted from your total earnings).

Please note that the experiment will only continue from one phase to another after everybody has pressed OK/PROCEED. For this reason, please press OK/PROCEED as soon as you have made your decision.

PAYOFFS WHEN THE CHOOSER ACCEPTS THE PROPOSAL

When the Chooser accepts your proposal, your payoff is 60 minus 0.4 times the proposal:

Your payoff $=60-0.4 *$ proposal. 
When the Chooser accepts your proposal, the Chooser will receive a payoff of 60 minus the distance between her or his preferred outcome and the proposal:

Payoff Chooser $=60-$ distance(her or his preferred outcome and proposal).

It is possible for a Chooser to reject a proposal.

PAYOFFS WHEN THE CHOOSER REJECTS THE PROPOSAL

When the Chooser rejects a proposal, then the outcome is the status quo. In this case, you will receive 0 points and the Chooser will receive 0 points.

Notice that accepting an offer gives the Chooser a higher payoff than rejecting it if and only if the distance between the proposal and her preferred outcome is smaller than 60. Your payoff is higher when the Chooser accepts than when she or he rejects in all cases.

EXAMPLE 1. Suppose the Chooser's preferred outcome turns out to be 80 (which you cannot know) and you make a proposal of 100 . Then, the distance between her preferred outcome and your proposal is $100-80=20$.

If the Chooser accepts, your payoff is $60-0.4 * 100=20$. The Chooser's payoff in this case is 60 $-20=40$.

If the Chooser rejects, your payoff is 0 and the Chooser's payoff is 0 .

EXAMPLE 2. Suppose the Chooser's preferred outcome turns out to be 80 and you make a proposal of 10. Then, the distance between her preferred outcome and your proposal is $80-10=$ 70.

If the Chooser accepts, your payoff is $60-0.4 * 10=56$. The Chooser's payoff in this case is 60 $-70=-10$.

If the Chooser rejects, your payoff is 0 and the Chooser's payoff is 0 .

\section{HISTORY OVERVIEW}

When making a decision, you may use the History Overview, which fills the lower part of the screen. The History Overview summarizes the results of the most recent 15 periods. (If less than 15 periods have been completed, this history overview contains results of all completed periods.) Apart from your own results in the previous periods, the history overview also contains the results of the other Chooser/Proposer pairs in your matching group. In total you are thus informed about the past results of the same group of five Chooser/Proposer pairs. All Choosers and Proposers in your matching group will have the same information. The presentation of information is different for Choosers than for Proposers.

\section{TABLE}

Below you see an example of the history overview. THE NUMBERS IN THE HISTORY OVERVIEW DO NOT INDICATE WHAT YOU SHOULD DO IN THE EXPERIMENT. The left part of the history overview is a Table with four columns. The first column labelled SUGGESTION contains the suggestions made by the Choosers in the recent previous periods. The second column labelled PREFERRED OUTCOME shows the preferred outcome of the Chooser. The third column labelled PROPOSAL gives the proposal that was made by the Proposer as a response to the suggestion in the same row. The fourth column labelled ACCEPTANCE shows whether the Chooser accepted or rejected the proposal. 


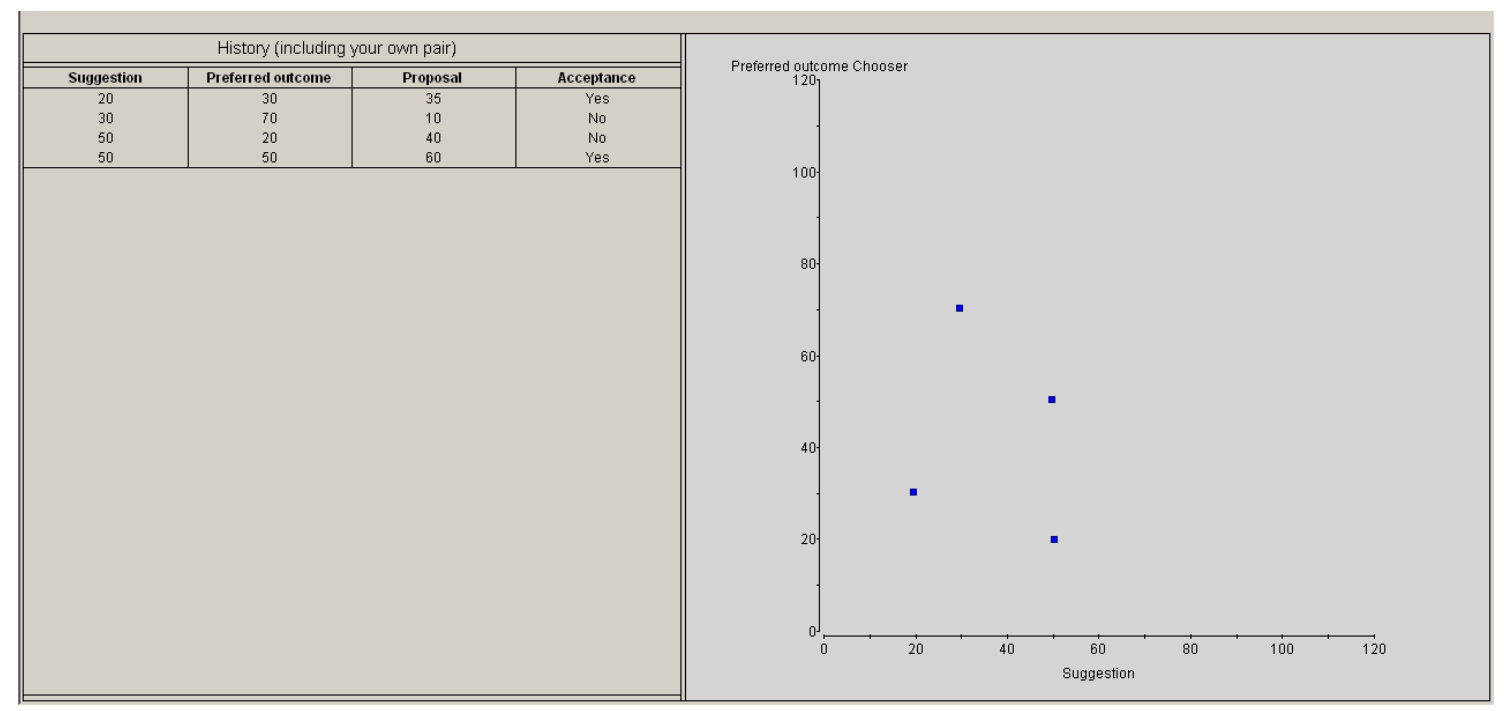

The results shown in the history overview will be sorted on the basis of suggestion in ascending order. (The lower the suggestion, the higher the place in the table.) When the suggestion is the same for two or more different results, these observations will be sorted on the basis of preferred outcome, again in ascending order. In the example above, this applies to the third and the fourth row, where two Choosers chose the same suggestion but had different preferred outcomes. More generally, observations have been sorted first on suggestion, then on preferred outcome, then on proposal and finally on acceptance or rejection.

\section{GRAPH}

On the right of the history overview, the most recent results are represented in a graph. The horizontal axis presents the suggestion and the vertical axis presents the proposal. Each previous observation is represented by a square. On the horizontal axis you can read the value of the suggestion for a particular result and on the vertical axis you can read the value of the corresponding proposal. If the square is green, the particular proposal was accepted and if the square is red with white inside, the particular proposal was rejected. (Choosers will see proposals on the vertical axis.)

EXAMPLE 1. Consider the square that is displayed in the lower left corner of the Graph shown above. Here, the Chooser made a suggestion of 20. This Chooser's preferred outcome was 30 .

You have now reached the end of the instructions. The next page contains some questions concerning the experiment. When all participants have answered all questions correctly, we will proceed with the experiment. 


\section{QUESTIONS}

Please answer the following questions. THE VALUES USED IN SOME QUESTIONS DO NOT INDICATE WHAT YOU SHOULD DO IN THE EXPERIMENT. RATHER, THEY HAVE BEEN CHOSEN TO FACILITATE CALCULATIONS.

1. Is the following statement correct? 'In each period I am coupled with the same Chooser.'

2. Is the following statement correct? 'I will observe the Chooser's preferred position before I make my proposal.'

3.

(A) What is the highest value the preferred outcome of a Chooser can take on?

(B) What is the highest value a suggestion of a Chooser can take on?

(C) What is the highest value a proposal of yours can take on?

4. Consider a period in which the Chooser's preferred outcome is 50. The Chooser chose to send a suggestion of 40 . You made a proposal of 20, which was accepted by the Chooser.

(A) What are your own earnings in this period?

(B) How much does the Chooser to whom you are paired earn?

5. Consider a period in which the Chooser's preferred outcome is 90. The Chooser chose to send a suggestion of 100 . You made a proposal of 0 , which was accepted by the Chooser.

(A) What are your own earnings in this period?

(B) How much does the Chooser to whom you are paired earn?

6. Consider a period in which the Chooser's preferred outcome is 30. The Chooser chose to send a suggestion of 40 . You made a proposal of 10 , which was rejected by the Chooser.

(A) What are your own earnings in this period?

(B) How much does the Chooser to whom you are paired earn?

When you are ready answering the questions, please raise your hand. 